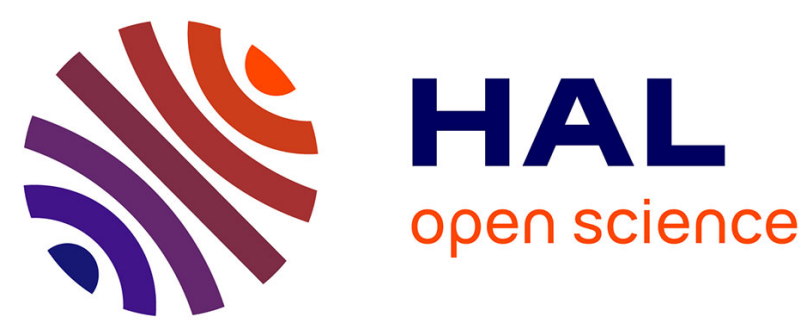

\title{
Highly emissive layers based on organic/inorganic nanohybrids using Aggregation Induced Emission effect
}

Jonathan Phelipot, Nicolas Ledos, Thomas Dombray, Matthew P Duffy, Mathieu Denis, Ting Wang, Yahia Didane, Meriem Gaceur, Qinye Bao, Xianjie Liu, et al.

\section{To cite this version:}

Jonathan Phelipot, Nicolas Ledos, Thomas Dombray, Matthew P Duffy, Mathieu Denis, et al.. Highly emissive layers based on organic/inorganic nanohybrids using Aggregation Induced Emission effect. Advanced Materials Technologies, 2022, 7 (1), pp.2100876. 10.1002/admt.202100876 . hal-03341916

\author{
HAL Id: hal-03341916 \\ https://hal.science/hal-03341916
}

Submitted on 13 Sep 2021

HAL is a multi-disciplinary open access archive for the deposit and dissemination of scientific research documents, whether they are published or not. The documents may come from teaching and research institutions in France or abroad, or from public or private research centers.
L'archive ouverte pluridisciplinaire $\mathbf{H A L}$, est destinée au dépôt et à la diffusion de documents scientifiques de niveau recherche, publiés ou non, émanant des établissements d'enseignement et de recherche français ou étrangers, des laboratoires publics ou privés. 


\section{Highly emissive layers based on organic/inorganic nanohybrids using Aggregation Induced Emission effect}

Jonathan Phelipot, ${ }^{1}$ Nicolas Ledos, ${ }^{2}$ Thomas Dombray, ${ }^{2}$ Matthew P. Duffy, ${ }^{2}$ Mathieu Denis, ${ }^{2}$ Ting Wang, ${ }^{1}$ Yahia Didane, ${ }^{1}$ Meriem Gaceur, ${ }^{1}$ Qinye Bao, ${ }^{3}$ Xianjie Liu, ${ }^{3}$ Mats Fahlman, ${ }^{3}$ Pietro Delugas, ${ }^{4}$ Alessandro Mattoni,${ }^{4}$ Denis Tondelier, ${ }^{5}$ Bernard Geffroy, ${ }^{5,6}$ Pierre-Antoine Bouit, ${ }^{2}$ Olivier Margeat, ${ }^{1, *}$ Jörg Ackermann, ${ }^{1}$ Muriel Hissler ${ }^{2}, *$

1. J. Phelipot, Dr. T. Wang, Dr. Y. Didane, Dr. M. Gaceur, Dr. O. Margeat, Dr. J. Ackermann.

Aix Marseille Univ, CNRS, CINaM, Marseille, France.

E-mail: olivier.margeat@univ-amu.fr

2. N. Ledos, Dr. T. Dombray, Dr. M.P. Duffy, Dr. M. Denis, Dr. P-A. Bouit, Prof. Dr. M. Hissler.

Univ Rennes, CNRS, ISCR - UMR 6226, F-35000 Rennes, France.

E-mail: muriel.hissler@univ-rennes1.fr

3. Dr. Q. Bao, Dr. X. Liu, Prof. Dr. M. Fahlman.

Laboratory for Organic Electronics, ITN, Linköping University, Norrköping, Sweden.

4. Dr. P. Delugas, Dr. A. Mattoni.

Istituto Officina dei Materiali (CNR - IOM) Cagliari, Cittadella Universitaria, I-09042

Monserrato (Ca), Italy.

5. Dr. D. Tondelier, Dr. B. Geffroy.

Laboratoire de Physique des Interfaces et des Couches Minces (LPICM), CNRS, Ecole Polytechnique, IP Paris, Palaiseau Cedex, France.

6. Université Paris-Saclay, CEA, CNRS, NIMBE, LICSEN, 91191, Gif-sur-Yvette, France.

Keywords: hybrid materials, AIE, fluorescence, nanoparticles, hydroxyoxoposphole

Abstract. Fluorescent nanohybrids, based on $\pi$-extended hydroxyoxophosphole ligands grafted onto $\mathrm{ZnO}$ nanoparticles, were designed and studied. The restriction of the intramolecular motions of the organic fluorophore, through either aggregates' formation in solution or processing into thin films, forms highly emissive materials due to a strong Aggregation Induced Emission effect. Theoretical calculations and XPS analyses were performed to analyze the interactions between the organic and inorganic counterparts. Preliminary results on the use of these nanohybrids as solution-processed emissive layers in Organic Light Emitting Diodes (OLEDs) illustrate their potential for lighting applications. 


\section{Introduction}

Light-emitting materials are nowadays important components in a large field of applications such as light-emitting diodes (LEDs), ${ }^{[1]}$ security, ${ }^{[1]}$ biology ${ }^{[2]}$ and nano-medicine. ${ }^{[3]}$ For many applications, generation of highly emissive nanoparticles has opened new opportunities for future technologies such as QD-LEDs, lasers and bio-labelling. ${ }^{[4-6]}$ Nano- and microscale materials with tailored emission properties have been developed with rationally designed organic molecules. ${ }^{[7-9]}$ While these organic nanoparticles show strong emission, control over size, shape, distribution and processing remains challenging. ${ }^{[7-9]}$ One possibility to enhance the emission of organic fluorophores and to control the size of emissive nanoparticles at the same time is their grafting onto inorganic nanoparticle surfaces. For example, the coupling of fluorophore molecules with the plasmon resonance of metallic nanoparticles could enhance their emission. ${ }^{[10,11]}$ Electronic interaction between the ligand molecule and the inorganic nanocore was found to form new emissive excited states of the ligands. ${ }^{[12]}$ Thus, fluorescent nanohybrids are a fascinating new class of materials generating new or improved optical properties in a synergetic way from both organic ligand and inorganic nanoparticles. However, in the objective of generating highly emissive hybrid nanoparticles, the grafting of organic ligands onto inorganic nanoparticle surfaces often to poor emission enhancement. ${ }^{[13]}$ Indeed, many planar organic luminogens tend to aggregate due to strong $\pi-\pi$ interaction, which usually turns off light emission. As result, high concentration of ligands on the nanoparticle surface, needed to generate strong light emission, leads to aggregation-caused quenching phenomena (ACQ) ${ }^{[14,15]}$ To prevent such quenching, molecular design offers a very elegant strategy to avoid detrimental intermolecular interaction and generates the contrary effect of ACQ, which refers to aggregation-induced emission (AIE) processes. ${ }^{[14]}$ Once the molecules form aggregates, for example in thin films or in a crystal, strong fluorescence enhancement is observed, due to the restriction of their intramolecular rotations in the aggregate state. ${ }^{[14]}$ The AIE phenomenon is often based on the use of nonplanar propeller-shaped rotor-like structures. 
Such simplified picture works nicely with propeller-like derivatives such polyphenyl-siloles, phospholes or tetraphenylethyelene. However, the photophysics of solid-state luminophore is more complex than this simple AIE / ACQ duality. ${ }^{[16,17]}$ Recently, a salicylaldehyde azine has been grafted onto colloidal $\mathrm{GaOOH} / \mathrm{H}_{2} \mathrm{O}$ nanocubes, ${ }^{[18]}$ generating strong hydrophobic interactions which effectively restrict the molecular motions and thus enhance the emission. Similar trends were observed with carbazole derivatives on various inorganic or metal nanocrystals. ${ }^{[19,20]}$ However, to be used in optoelectronic devices as an emitting layer, such organic-inorganic nanohybrid materials should provide suitable transport properties together with their optical emission, and be able to form thin films with optical quality. We thus envisaged to achieve such properties by using AIE ligands synergistically combined with semiconducting nanoparticles. In this work, we present the synthesis of AIE nanohybrids prepared via grafting organic AIE ligands onto high bandgap semiconducting nanoparticles. Zinc oxide $(\mathrm{ZnO})$ was chosen as the inorganic material for both its ability to be easily functionalized and its potential electron transport properties as n-type semiconductor. Among the AIE fluorophores, we selected phosphole-based luminophores. ${ }^{[21-27]}$ Hence, the phosphole stator allows accessing easily to hydroxyoxophosphole which can coordinate intimately onto metal oxide semiconductors. ${ }^{[28]}$ The structure-properties relationships leading to highly emissive materials have been investigated. Integration of these novel nanohybrids into solutionprocessed LED demonstrates that this strategy allows generating electroluminescent films.

\section{Results and Discussion}

\subsection{Nanohybrid syntheses and characterizations of the grafting}

The AIE nanohybrids were synthesized in solution by molecular grafting on the surface of $\mathrm{ZnO}$ nanospheres (diameter ca. $6 \mathrm{~nm}$ ) of one of the selected AIE ligands 1-3 (Figure 1a). ${ }^{[29,30]}$ In a typical synthesis in organic solvents such as chloroform, a solution containing a specific amount 
of $\mathrm{ZnO}$ nanoparticles is mixed with a solution containing an adequate concentration of one of the AIE ligands allowing to control the amount of grafted molecules per $\mathrm{ZnO}$ nanoparticle. Varying the concentration of ligands by keeping the amount of ZnO NPs constant is then used to produce AIE nanohybrids with different ratio of organic/inorganic composition. The scheme on Figure 1c represents the nanohybrid formation in solution and the resulting emissive materials, either aggregates in solution or thin film when spin-coated on a substrate.

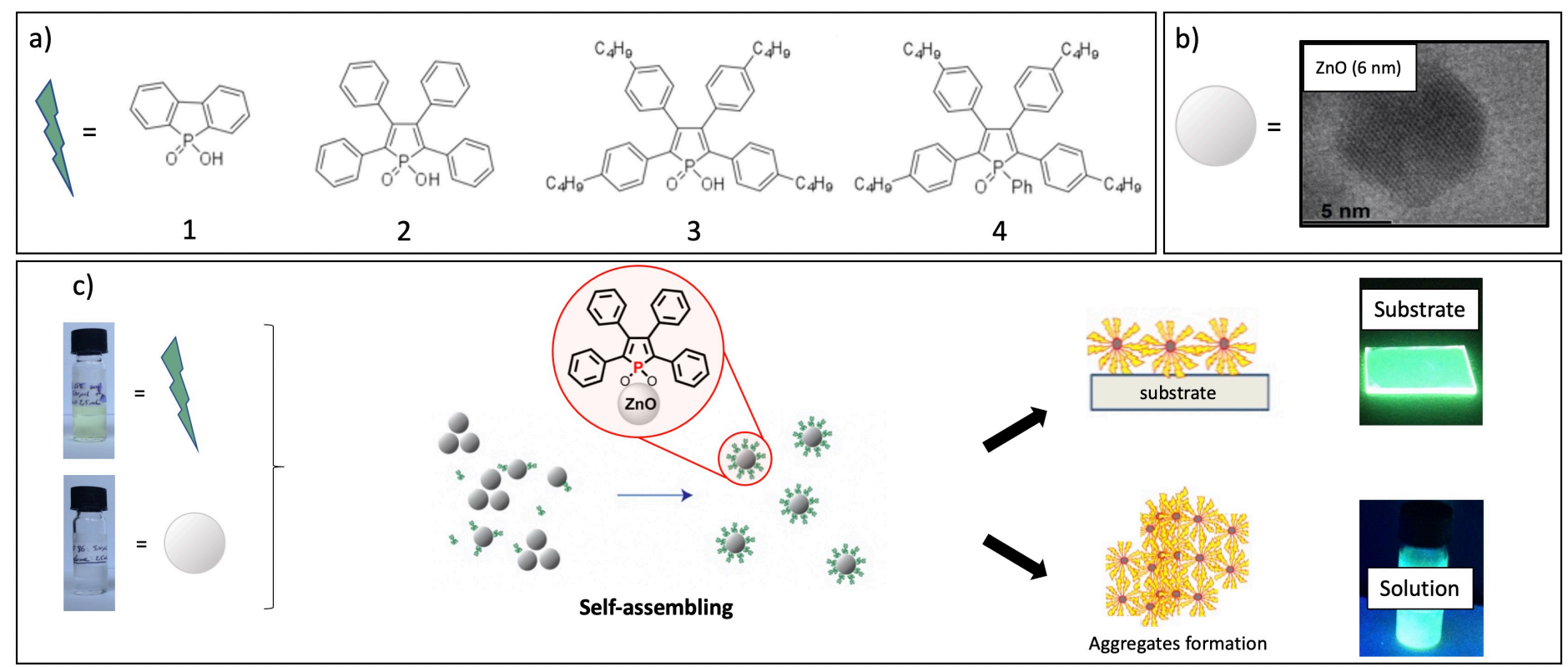

Figure 1. a) Molecular structures of the fluorescent molecules $(1,2,3)$ bearing phosphole unit to bind to $\mathrm{ZnO}$ surface; molecule $\mathbf{4}$ is a reference phosphole without grafting unit. b) Zinc oxide nanoparticles used for the study. c) Scheme of the AIE effect when molecules are grafted onto $\mathrm{ZnO}$ nanoparticles forming highly emissive materials, used as layer on a substrate or aggregates in solution.

The efficient grafting of the ligands 1, 2, 3 via the hydroxyoxophosphole group onto $\mathrm{ZnO}$ nanospheres was investigated by FTIR spectroscopy, XPS and theoretical calculations. The powder FTIR spectra of compounds $\mathbf{2}, \mathrm{ZnO}$ and nanohybrids $\mathrm{ZnO}: 2$ (recovered from solution by centrifugation) clearly demonstrates the occurrence of the grafting (Figure 2a). It can be seen that the band at $990 \mathrm{~cm}^{-1}$ corresponding to the $v(\mathrm{P}-\mathrm{OH})$ vibration of compound 2 disappears after grafting as expected, ${ }^{[28,31]}$ together with the removal of acetates from the $\mathrm{ZnO}$ 
surface (lower intensities for bands at 1440 and $1560 \mathrm{~cm}^{-1}$ ), ${ }^{[32]}$ while a new band at $1110 \mathrm{~cm}^{-1}$ appears that can be addressed to the $v(\mathrm{O}-\mathrm{P}-\mathrm{O})$ vibration. ${ }^{[28,33]}$
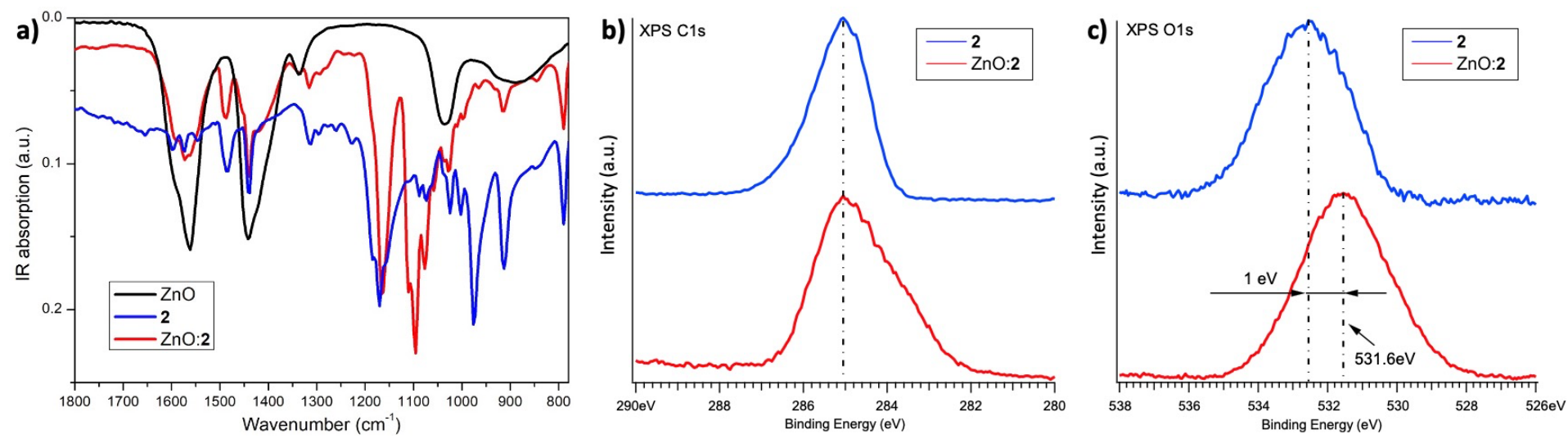

Figure 2. a) FTIR spectra of compounds 2, $\mathrm{ZnO}$ and $\mathrm{ZnO}$ :2. b-c) XPS spectra of compounds 2 and $\mathrm{ZnO} \mathbf{2}$.

Theoretical calculations were realized by Density Functional Theory (DFT) to study the different possible binding modes of $\mathbf{2}$ onto $\mathrm{ZnO}$ surfaces (see ESI for computational details). We focused in particular on the binding mode of hydroxyoxophosphole group exploring its bidentate, monodentate, protonated and deprotonated configurations. The results clearly indicate that the bidentate grafting mode is the most stable configuration for the binding of 2 onto the $\mathrm{ZnO}$ surface, with the proton spontaneously moving from the $\mathrm{POOH}$ group to the surface. The band alignment in the case of $\mathbf{2}$ bonded on the nonpolar (01-10) crystalline $\mathrm{ZnO}$ surface have been calculated (Figure S4). A downshift of the molecular levels is found with a weak charge displacement only visible at the O-Zn bond. Furthermore, the XPS analyses suggest the occurrence of charge transfer from the $\mathrm{ZnO}$ to the ligand $\mathbf{2}$ during grafting as a more electron rich environment in the molecule produces a shift towards lower binding energy in the XPS core level spectra. In details, the XPS analysis of nanohybrids ZnO:2 as well as of the nongrafted ligand 2 show a new shoulder for $\mathrm{C} 1 \mathrm{~s}$ peak at lower binding energy after grafting (Figures 2b-c, and wide scan on Figure S5). This shift is most likely due to charge transfer from the $\mathrm{ZnO}$ nanoparticle surface to carbon atoms. We further observe a shift towards lower 
binding energy for $\mathrm{O} 1 \mathrm{~s}$, though this shift can be addressed to the contribution from the $\mathrm{ZnO}$ oxygens. ${ }^{[34]}$

\subsection{Optical properties of nanohybrids in solution}

The absorption and emission spectra of the molecules 1-4 before and after adding $\mathrm{ZnO}$ in the chloroform solution were studied. Table S2 summarizes the optical parameters of the ligands. The ligands 2-4 exhibit $\pi-\pi^{*}$ absorption band centered around $380-410 \mathrm{~nm}$ in the UV-visible region (Figure 3 and S6) which is blue-shifted down to $280 \mathrm{~nm}$ for ligand 1 (Figure S7). As anticipated, compounds 2-4 present a very weak emission in solution centered around 500-530 nm while compound 1, as expected from its planar structure, displays in solution a higher emission at $360 \mathrm{~nm}$ (see Table S2). Then, the effects of their grafting on $\mathrm{ZnO}$ nanoparticles on the optical properties have been investigated. When the concentration of $\mathrm{ZnO}$ nanoparticles was increased (monitored by UV absorption - Figure S7b) in a chloroform solution of 1, a severe quenching of the characteristic emission of the ligand was observed (Figure S7c). The grafting of 1 onto $\mathrm{ZnO}$ nanoparticles leads indeed to strong ACQ effect due to the $\pi$ - $\pi$ stacking at the $\mathrm{ZnO}$ surface. ${ }^{[35]}$ Then finally, by continuing the addition of $\mathrm{ZnO}$, the classical $\mathrm{ZnO}$ defect emission at $550 \mathrm{~nm}$ gradually appeared. ${ }^{[36]}$

Interestingly, the grafting of ligands $\mathbf{2}$ and $\mathbf{3}$ showed a completely different behavior. Indeed, in these cases, an emission enhancement was observed. The mass ratio between $\mathrm{ZnO}$ nanoparticles and the organic compounds was optimized to generate the highest emission and was found equal to $\mathrm{ZnO}$ :ligand $=2: 1$. While the emission of ligand $\mathbf{2}$ is enhanced by a factor up to 500 by the injection of $\mathrm{ZnO}$ into the solution, ligand $\mathbf{3}$ only shows an emission enhancement in the range of 6-10 (Figures 3b/d). Additionally, there is a strong light scattering signal appearing in the absorption spectrum of $\mathrm{ZnO}: 2$ indicating the formation of large nanohybrids aggregates while grafting (Figure 3a). Transmission Electron Microscopy (TEM) 
analyses confirmed the presence of these tens to hundreds of nanometers large aggregates in solution (Figure S8). In contrast, grafting of $\mathbf{3}$ leads to nanohybrids $\mathrm{ZnO}: \mathbf{3}$ highly soluble in chloroform, as validated by DLS/TEM analyses and the absence of any noticeable scattering signal (Figure 3b). In the case of compound 4, the reference molecule without grafting unit, there is no change in absorption and emission introduced by the presence of $\mathrm{ZnO}$ nanoparticles (Figure S6). This indicates clearly that the optical changes observed for ligands $\mathbf{2}$ and $\mathbf{3}$ are directly related to their grafting onto $\mathrm{ZnO}$ nanoparticle surfaces. Due to the fact that both ligands $\mathbf{2}$ and $\mathbf{3}$ are composed of the same hydroxyoxophosphole grafting unit and only differ by the presence of long alkyl chains in the case of $\mathbf{3}$, it can be supposed that the much higher emission enhancement in solution in the case of $\mathrm{ZnO}: 2$ compare to $\mathrm{ZnO}: 3$ is related to the restriction of phenyl ring rotation through the interactions between nanohybrids inside the large aggregates.
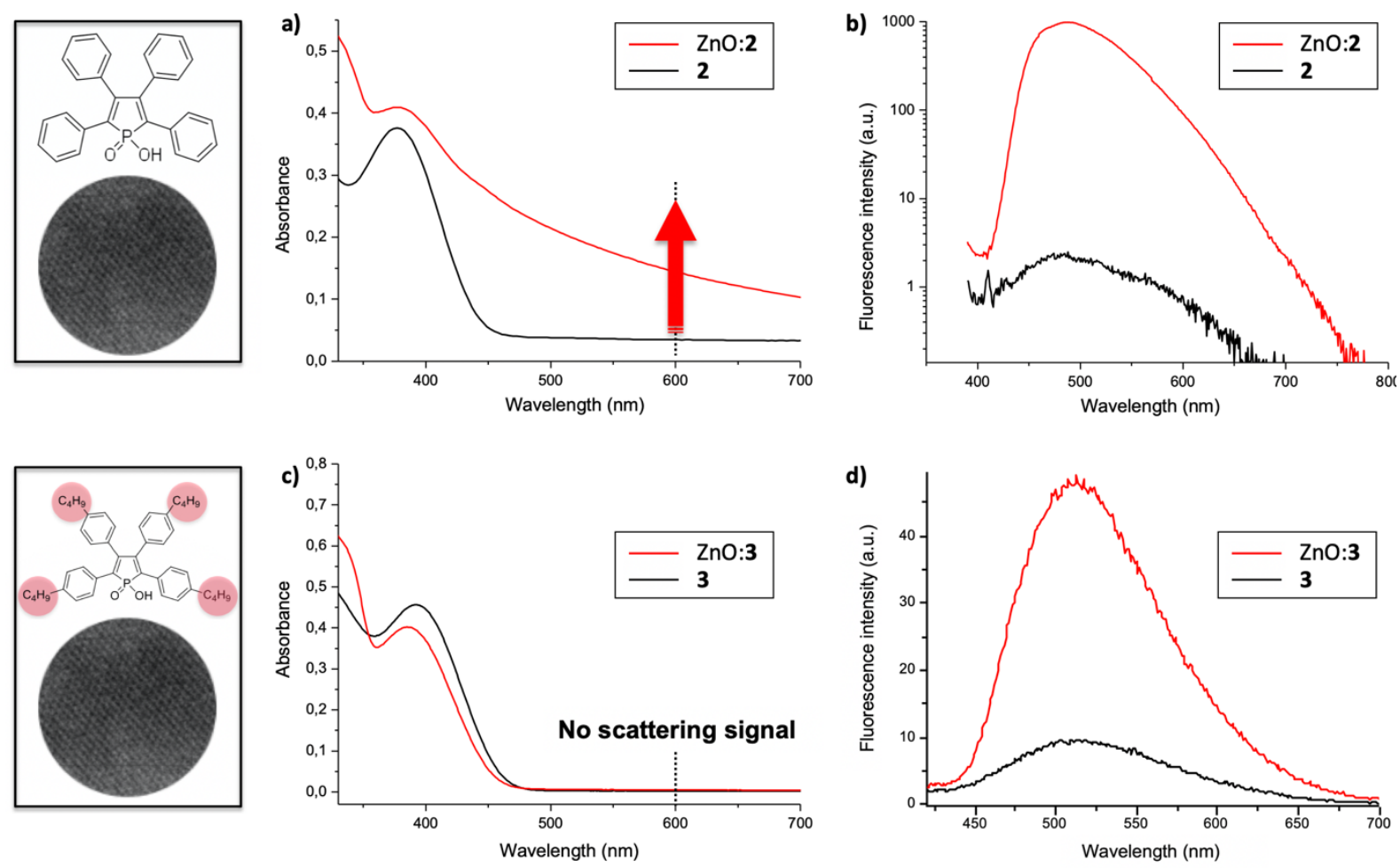

Figure 3. Top: absorption spectra (a) and emission spectra at $\lambda_{\mathrm{ex}}=380 \mathrm{~nm}$ (b) of $\mathbf{2}$ and $\mathrm{ZnO}: \mathbf{2}$ in chloroform solution. Bottom: absorption spectra (c) and emission spectra at $\lambda_{\mathrm{ex}}=380 \mathrm{~nm}$ (d) of $\mathbf{3}$ and $\mathrm{ZnO}: \mathbf{3}$ in chloroform solution. 
In order to deeper understand the grafting process and the induction of strong AIE effect, the time evolution of the luminescence spectra was studied for the nanohybrids $\mathrm{ZnO}: 2$ and $\mathrm{ZnO}: 3$ right after injection of the $\mathrm{ZnO}$ nanoparticles in solution. Simultaneously, the time evolution of their absorption spectra was recorded at $600 \mathrm{~nm}$, i.e., at a wavelength without ligand absorption but well located to measure the optical signal related to light scattering upon aggregates formation (Figure 4). Dynamic light scattering (DLS) measurements helped to characterize the initial $\mathrm{ZnO}$ solution, especially to determine that $\mathrm{ZnO}$ nanoparticle clusters about $15 \mathrm{~nm}$ large were present in this starting solution. Taking into account that the size of the $\mathrm{ZnO}$ nanoparticles is $6 \mathrm{~nm}( \pm 1 \mathrm{~nm})$, there are small clusters composed of few nanoparticles already present in solution before grafting. After injection of $\mathrm{ZnO}$ nanoparticles, both nanohybrids $\mathrm{ZnO} 2 \mathrm{2}$ and $\mathrm{ZnO}: 3$ showed first a weak decrease in light scattering during the first minutes, indicating that the grafting of the molecules weakly reduced the cluster size of $\mathrm{ZnO}$ nanoparticles. In the case of nanohybrids $\mathrm{ZnO}: 3$, the light scattering signal remained then stable thanks to the solubility of these nanohybrids in solution, whereas the fluorescence intensity increased to reach the stable emission enhancement in a factor of 6-10. This moderate increase can be rationalized on the basis of the chemical modification of the fluorophore upon grafting (loss of the O-H vibrator). Interestingly, in the case of nanohybrids $\mathrm{ZnO}$ :2, a different behavior with two phases was observed. During the first 15 minutes (see vertical dotted line as eye guide in Figure 4a), the first phase was similar for both nanohybrids as the fluorescence intensity of $\mathrm{ZnO}: 2$ increased by the same typical factor of 8-12. After these 15 minutes, during the second phase, the scattering signal started to increase strongly and saturated in intensity after 30 minutes, indicating the formation of $\mathrm{ZnO} 22$ nanohybrids large aggregates. The corresponding fluorescence intensity increased simultaneously very fast by another factor of 10 with the large aggregates' formation. The later emission enhancement during this second phase can be directly 
addressed to the restriction of phenyl ring rotation during the large aggregates' formation, i.e., the AIE effect.
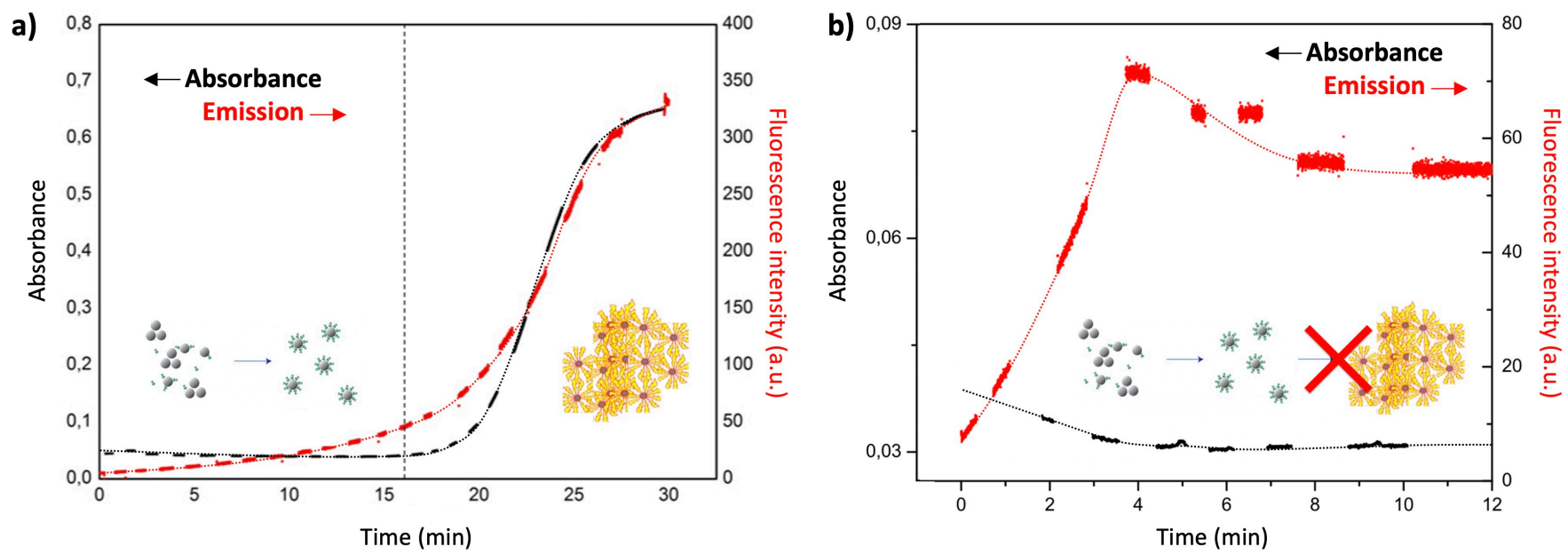

Figure 4. Time resolved absorbance measured at $600 \mathrm{~nm}$ (black lines) and fluorescence intensity (red lines) of $\mathrm{ZnO}: 2$ (a) and $\mathrm{ZnO}: 3$ (b) nanohybrids. Zero minute corresponds to the time when $\mathrm{ZnO}$ was injected into molecules solution in chloroform.

\subsection{Thin film photoluminescence and electroluminescence}

In order to evaluate these novel nanomaterials for lighting applications, we studied the optical properties of $\mathrm{ZnO}: 2$ and $\mathrm{ZnO}: 3$ nanohybrids in thin films. As $\mathrm{ZnO}: 2$ nanohybrids form large aggregates in solution, their solution processing via spin coating onto glass substrates leads to films of poor optical quality. Very high roughness and only partial surface coverage were indeed determined by Atomic Force Microscopy, thus making these nanohybrids non suitable for LED fabrication. In contrast, nanohybrids $\mathrm{ZnO}: 3$, thanks to their high solubility, allowed the processing of very smooth $(\mathrm{RMS}$ roughness $=1.3 \mathrm{~nm})$ and closely packed homogeneous layers on glass (Figures 5a/b). The layers based on $\mathrm{ZnO} \mathbf{2}$ are strongly emissive with quantum yields (QY) yield up to $38 \%$ as it was expected from the analyses done in solution. Importantly, we found out that also nanohybrids $\mathrm{ZnO}: 3$ formed layers with high emission (Figure 5c) with QY up to $39 \%$, that is strongly increased compared to the free ligand measured in the same 
conditions $(\phi=5 \%)$ and more importantly, nearly identical to the emission of $\mathrm{ZnO}: 2$ layers.

This result shows clearly that ligand $\mathbf{3}$, even grafted onto $\mathrm{ZnO}$ nanoparticles, still owns intramolecular rotation of phenyl rings in solution, but processing into thin films definitely freezes phenyl rotations via intermolecular interactions and thus generates strong AIE effect.

a)

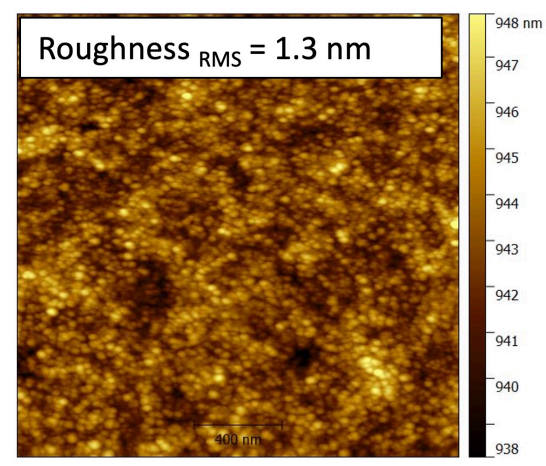

b)

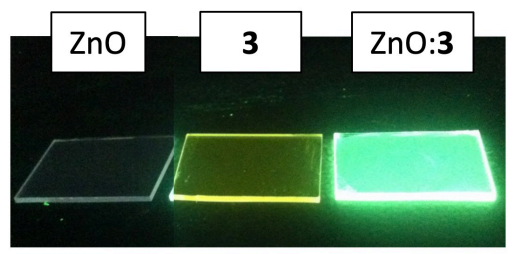

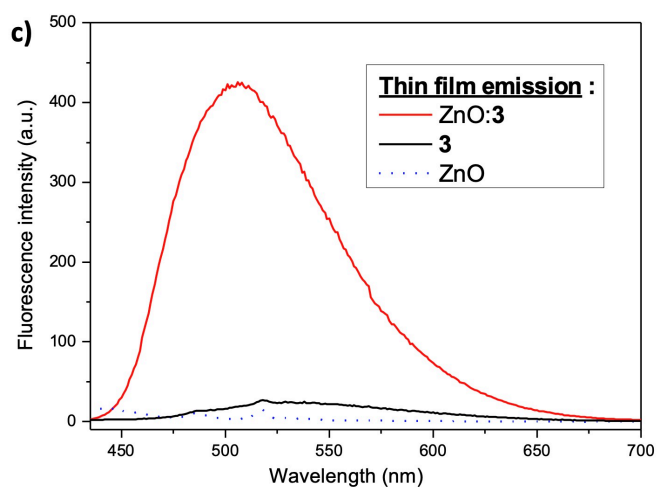

Figure 5. a) AFM image of $\mathrm{ZnO}: 3$ thin film spin-coated on glass substrate. b) Photograph image under UV-light of $\mathrm{ZnO}, 3$ and nanohybrids $\mathrm{ZnO}: \mathbf{3}$ spin-coated thin films. c) Fluorescence spectra of $\mathrm{ZnO}, \mathbf{3}$ and nanohybrids $\mathrm{ZnO}: 3$ thin films at $\lambda_{\mathrm{ex}}=380 \mathrm{~nm}$.

While $\mathrm{ZnO}: 2$ films were not suitable for device application due to their poor quality, thanks to the high optical quality of nanohybrids $\mathrm{ZnO}: 3$ thin films, the electroluminescent properties of this material were studied as light-emitting layer in simple LED structures. The device structure (Figure S9) consists of ITO/CuPc/HTL/ $\mathrm{ZnO}: 3 / \mathrm{mCP} / \mathrm{Alq}_{3} / \mathrm{LiF} / \mathrm{Al}$ with $\mathrm{CuPc}$ as hole injection layer and $\mathrm{mCP}$ as hole blocking layer to avoid emission from $\mathrm{Alq}_{3}$. The hole transport layer (HTL) is a $20 \mathrm{~nm}$ thick film of polyvinylcarbazole used to reduce the leakage current within the device. The emitting layer $\mathrm{ZnO}: 3$ is solution-processed on the stack from concentrated nanohybrids solution (see ESI for details on LED fabrication). The current density-voltageluminance (J-V-L) curves of the diode (Figure 6a) shows a threshold voltage near $4 \mathrm{~V}$ with a maximum emission at $90 \mathrm{~cd} / \mathrm{m}^{2}$ for a current density of $100 \mathrm{~mA} / \mathrm{cm}^{2}$. Inset shows two photograph images of the diode working at 30 and $50 \mathrm{~mA} / \mathrm{cm}^{2}$, respectively, having homogeneous emission over the whole emitting surface $\left(0.28 \mathrm{~cm}^{2}\right)$. The electroluminescence 
spectrum corresponds to the photoluminescence spectrum of nanohybrid $\mathrm{ZnO}: 3$ (maximum at $514 \mathrm{~nm}$ ) proving that the emission comes from the nanohybrid layer (Figure 6b). These results show that this new class of nanohybrid material is able to act as emissive layer in solutionprocessed LED devices, even though the moderate luminance and its saturation at high current densities deserve optimizations in future work.
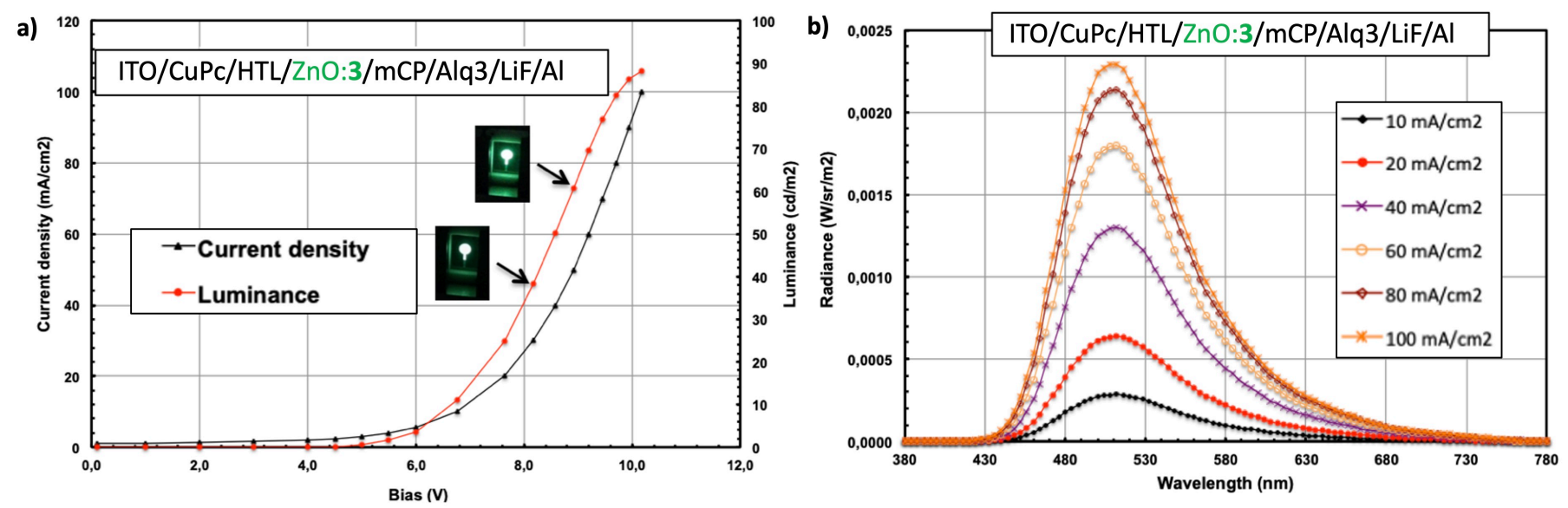

Figure 6. a) Electroluminescent J-V-L curves of the LED devices. b) Radiance spectra of the LED devices as a function of current density.

\section{Conclusion}

To summary, novel fluorescent nanohybrids, based on $\pi$-extended hydroxyoxophosphole ligands grafted onto $\mathrm{ZnO}$ surface, were designed and studied. Furthermore, the restriction of the intramolecular motions of the organic fluorophore, through either aggregates' formation in solution or processing into thin films, is found to form highly emissive materials due to a strong AIE effect. Theoretical calculations and XPS analysis show that electronic coupling between the ligands and the $\mathrm{ZnO}$ nanoparticles occurs leading to partial charge transfer to the ligand from the $\mathrm{ZnO}$ core. Preliminary results on the use of these nanohybrids as solution-processed emissive layers for lighting applications indicate high quantum efficiencies in the thin films allowing electroluminescence. Future work will explore the potential of these nanohybrids 
using more efficient charge transport layers together with multicolor highly emissive graftable molecules leading to major advances compared to pure organic materials or inorganic nanoparticles for lighting devices or optical detections at nanoscale.

\section{Experimental Section/Methods}

All experiments were performed under an atmosphere of dry argon using standard Schlenk techniques. Commercially available reagents were used as received without further purification. Solvents were freshly purified using MBRAUN SPS-800 drying columns. Separations were performed by gravity column chromatography on basic alumina (Aldrich, Type 5016A, 150 mesh, $58 \AA$ ) or silica gel (Merck Geduran $60,0.063-0.200 \mathrm{~mm}$ ). ${ }^{1} \mathrm{H},{ }^{13} \mathrm{C}$, and ${ }^{31} \mathrm{P}$ NMR spectra were recorded on Bruker AV III 300 and $400 \mathrm{MHz}$ NMR spectrometers equiped with BBO or BBFO probeheads. Assignment of proton and carbon atoms is based on COSY, NOESY, edited-HSQC and HMBC experiments. ${ }^{1} \mathrm{H}$ and ${ }^{13} \mathrm{C}$ NMR chemical shifts were reported in parts per million (ppm) using residual solvent signal as reference. High-resolution mass spectra were obtained on a Varian MAT 311 or ZabSpec TOF Micromass instrument at CRMPO (Scanmat, UMS 2001). UV-Visible spectra were recorded at room temperature on a VARIAN Cary 5000 spectrophotometer. The UV-Visemission and excitation spectra measurements were recorded on a FL 920 Edimburgh Instrument equipped with a Hamamatsu R5509-73 photomultiplier for the NIR domain (300-1700 nm) and corrected for the response of the photomultiplier. Quantum yields in solution were calculated relative to quinine sulfate $\left(\mathrm{H}_{2} \mathrm{SO}_{4}, 0.1 \mathrm{M}\right), \phi_{\text {ref }}=0.55$. Detailed

syntheses and NMR characterizations of organic compounds are given in Supporting Information.

\section{Supporting Information}

Supporting Information is available from the Wiley Online Library or from the author. 


\section{Acknowledgements}

This work is supported by the Ministère de la Recherche et de l'Enseignement Supérieur, the CNRS, the Région Bretagne (ARED grant to NL), the French National Research Agency (ANR Fluohyb ANR-17-CE09-0020) and the SATT Sud-Est. The authors thank T. Roisnel (CDifX, ISCR) for X-ray diffraction analysis and A.Ranguis (CINaM) for AFM measurements. A.M. acknowledges MUR for project PON04a2 00490 M2M Netergit and PRACE access to Marconi KNL at CINECA, Italy, through project PROVING-IL (2019204911).

Received: ((will be filled in by the editorial staff))

Revised: ((will be filled in by the editorial staff)) Published online: ((will be filled in by the editorial staff))

\section{References}

[1] W. Yao, Q. Tian, W. Wu, Adv. Opt. Mater. 2019, 7, 1801171.

[2] E. Thimsen, B. Sadtler, M. Y. Berezin, Shortwave-infrared (SWIR) emitters for biological imaging: A review of challenges and opportunities, Vol. 6, Walter de Gruyter GmbH, 2017, pp. 1043-1054.

[3] J. Song, J. Qu, M. T. Swihart, P. N. Prasad, Nanomedicine Nanotechnology, Biol. Med. 2016, 12,771 .

[4] B. S. Mashford, M. Stevenson, Z. Popovic, C. Hamilton, Z. Zhou, C. Breen, J. Steckel, V. Bulovic, M. Bawendi, S. Coe-Sullivan, P. T. Kazlas, Nat. Photonics 2013, 7, 407.

[5] D. I. Son, B. W. Kwon, D. H. Park, W.-S. Seo, Y. Yi, B. Angadi, C.-L. Lee, W. K. Choi, Nat. Nanotechnol. 2012, 7, 465.

[6] U. Resch-Genger, M. Grabolle, S. Cavaliere-Jaricot, R. Nitschke, T. Nann, Nat. 
Methods 2008, 5, 763.

[7] Y.-T. Tsai, K.-P. Tseng, Y.-F. Chen, C.-C. Wu, G.-L. Fan, K.-T. Wong, G. Wantz, L. Hirsch, G. Raffy, A. Del Guerzo, D. M. Bassani, ACS Nano 2016, 10, 998.

[8] E. Genin, Z. Gao, J. A. Varela, J. Daniel, T. Bsaibess, I. Gosse, L. Groc, L. Cognet, M. Blanchard-Desce, Adv. Mater. 2014, 26, 2258.

[9] D. Kim, K. Jeong, J. E. Kwon, H. Park, S. Lee, S. Kim, S. Y. Park, Nat. Commun. 2019, 10, 3089.

[10] S. Kühn, U. Håkanson, L. Rogobete, V. Sandoghdar, Phys. Rev. Lett. 2006, 97.

[11] F. Tam, G. P. Goodrich, B. R. Johnson, N. J. Halas, Nano Lett. 2007, 7, 496.

[12] X. Cai, R. M. Adhikari, K. C. Anyaogu, S. S. Palayangoda, L. A. Estrada, K. De Puran, D. C. Neckers, J. Am. Chem. Soc. 2009, 131, 1648.

[13] U. Jabeen, T. Adhikari, S. M. Shah, S. U. Khan, D. Pathak, J. M. Nunzi, Opt. Mater. 2018, $83,165$.

[14] Y. Hong, J. W. Y. Lam, B. Z. Tang, Chem. Commun. 2009, 4332.

[15] Z. Zhao, H. Zhang, J. W. Y. Lam, B. Z. Tang, Angew. Chemie Int. Ed. 2020, 59, 9888.

[16] F. Würthner, T. E. Kaiser, C. R. Saha-Möller, Angew. Chemie Int. Ed. 2011, 50, 3376.

[17] J. Shi, L. E. Aguilar Suarez, S.-J. Yoon, S. Varghese, C. Serpa, S. Y. Park, L. Lüer, D. Roca-Sanjuán, B. Milián-Medina, J. Gierschner, J. Phys. Chem. C 2017, 121, 23166.

[18] T. Zheng, J.-L. Xu, X.-J. Wang, J. Zhang, X. Jiao, T. Wang, D. Chen, Chem. Commun. 2016, 52, 6922 .

[19] C. Li, X. Liu, M. Yuan, J. Li, Y. Guo, J. Xu, M. Zhu, J. Lv, H. Liu, Y. Li, Langmuir 2007, 23, 6754

[20] P. Kumar De, D. C. Neckers, Photochem. Photobiol. Sci. 2013, 12, 363.

[21] F. Bu, E. Wang, Q. Peng, R. Hu, A. Qin, Z. Zhao, B. Z. Tang, Chem. - A Eur. J. 2015, $21,4440$.

[22] M. P. Duffy, W. Delaunay, P.-A. Bouit, M. Hissler, Chem. Soc. Rev. 2016, 45, 5296. 
[23] Y. Ren, T. Baumgartner, Dalt. Trans. 2012, 41, 7792.

[24] K. Shiraishi, T. Kashiwabara, T. Sanji, M. Tanaka, New J. Chem. 2009, 33, 1680.

[25] A. Fukazawa, Y. Ichihashi, S. Yamaguchi, New J. Chem. 2010, 34, 1537.

[26] F. Riobé, R. Szűcs, P.-A. Bouit, D. Tondelier, B. Geffroy, F. Aparicio, J. Buendía, L. Sánchez, R. Réau, L. Nyulászi, M. Hissler, Chem. - A Eur. J. 2015, 21, 6547.

[27] P. Bolle, Y. Chéret, C. Roiland, L. Sanguinet, E. Faulques, H. Serier-Brault, P.-A. Bouit, M. Hissler, R. Dessapt, Chem. - An Asian J. 2019, 14, 1642.

[28] A. Kira, Y. Shibano, S. Kang, H. Hayashi, T. Umeyama, Y. Matano, H. Imahori, Chem. Lett. 2010, 39, 448.

[29] L. D. Freedman, B. R. Ezzel, R. N. Jenkins, R. M. Harris, Phosphorus 1974, 4, 199.

[30] J. Rabah, A. Escola, O. Jeannin, P.-A. Bouit, M. Hissler, F. Camerel, Chempluschem 2020, 85,79 .

[31] B. Zhang, T. Kong, W. Xu, R. Su, Y. Gao, G. Cheng, Langmuir 2010, 26, 4514.

[32] M. Estruga, C. Domingo, J. A. Ayllón, J. Mater. Chem. 2011, 21, 4408.

[33] D. Liu, W. Wu, Y. Qiu, S. Yang, S. Xiao, Q. Q. Wang, L. Ding, J. Wang, Langmuir 2008, 24,5052 .

[34] Q. Bao, X. Liu, Y. Xia, F. Gao, L.-D. Kauffmann, O. Margeat, J. Ackermann, M. Fahlman, J. Mater. Chem. A 2014, 2, 17676.

[35] C. Martini, G. Poize, D. Ferry, D. Kanehira, N. Yoshimoto, J. Ackermann, F. Fages, ChemPhysChem 2009, 10, 2465.

[36] Y. Gong, T. Andelman, G. F. Neumark, S. O’Brien, I. L. Kuskovsky, Nanoscale Res. Lett. 2007, 2, 297. 


\section{Table of Contents}

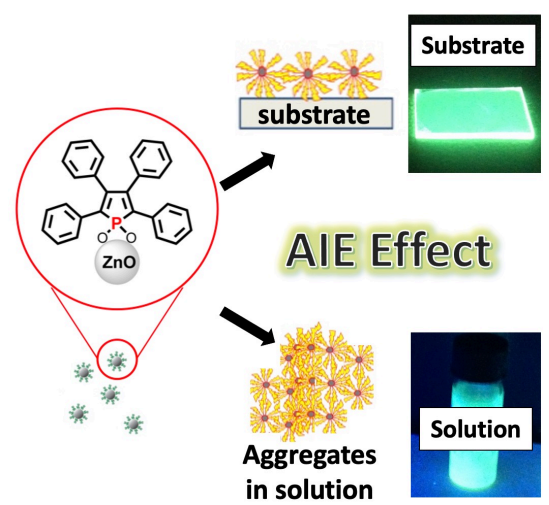

Novel fluorescent nanohybrids, based on $\pi$-extended hydroxyoxophosphole ligands grafted onto $\mathrm{ZnO}$ surface, were found to form highly emissive materials due to a strong Aggregation Induced Emission effect, through either aggregates' formation in solution or processing into thin films. The use of these nanohybrids as solution-processed emissive layers in OLEDs highlights their potential for lighting applications. 


\section{Supporting Information}

\section{Highly emissive layers based on organic/inorganic nanohybrids using Aggregation Induced Emission effect}

Jonathan Phelipot, ${ }^{1}$ Nicolas Ledos, ${ }^{2}$ Thomas Dombray, ${ }^{2}$ Matthew P. Duffy, ${ }^{2}$ Mathieu Denis, ${ }^{2}$ Ting Wang, ${ }^{1}$ Yahia Didane, ${ }^{1}$ Meriem Gaceur, ${ }^{1}$ Qinye Bao, ${ }^{3}$ Xianjie Liu, ${ }^{3}$ Mats Fahlman, ${ }^{3}$ Pietro Delugas, ${ }^{4}$ Alessandro Mattoni, ${ }^{4}$ Denis Tondelier, ${ }^{5}$ Bernard Geffroy, ${ }^{5,6}$ Pierre-Antoine Bouit, ${ }^{2}$ Olivier Margeat, ${ }^{1, *}$ Jörg Ackermann, ${ }^{1}$ Muriel Hissler ${ }^{2, *}$

1. J. Phelipot, Dr. T. Wang, Dr. Y. Didane, Dr. M. Gaceur, Dr. O. Margeat, Dr. J. Ackermann. Aix Marseille Univ, CNRS, CINaM, Marseille, France.

E-mail: olivier.margeat@,univ-amu.fr

2. N. Ledos, Dr. T. Dombray, Dr. M.P. Duffy, Dr. M. Denis, Dr. P-A. Bouit, Prof. Dr. M. Hissler. Univ Rennes, CNRS, ISCR - UMR 6226, F-35000 Rennes, France.

E-mail: muriel.hissler@univ-rennes1.fr

3. Dr. Q. Bao, Dr. X. Liu, Prof. Dr. M. Fahlman.

Laboratory for Organic Electronics, ITN, Linköping University, Norrköping, Sweden.

4. Dr. P. Delugas, Dr. A. Mattoni.

Istituto Officina dei Materiali (CNR - IOM) Cagliari, Cittadella Universitaria, I-09042 Monserrato (Ca), Italy.

5. Dr. D. Tondelier, Dr. B. Geffroy.

Laboratoire de Physique des Interfaces et des Couches Minces (LPICM), CNRS, Ecole Polytechnique, IP Paris, Palaiseau Cedex, France.

6. Université Paris-Saclay, CEA, CNRS, NIMBE, LICSEN, 91191, Gif-sur-Yvette, France.

Experimental part

NMR spectra

Crystallographic data and structure refinement parameters

Theoretical calculations

XPS

Optical properties

Transmission Electron Microscopy

Devices fabrication page $S 2$

page $S 4$

page $S 6$

page $\mathbf{S 9}$

page $S 13$

page $S 14$

page $S 16$

page $S 17$ 


\section{Experimental Part}

\section{Experimental Part General.}

All experiments were performed under an atmosphere of dry argon using standard Schlenk techniques. Commercially available reagents were used as received without further purification. Solvents were freshly purified using MBRAUN SPS-800 drying columns. Separations were performed by gravity column chromatography on basic alumina (Aldrich, Type 5016A, 150 mesh, $58 \AA$ ) or silica gel (Merck Geduran 60, 0.063-0.200 mm). ${ }^{1} \mathrm{H},{ }^{13} \mathrm{C}$, and ${ }^{31} \mathrm{P}$ NMR spectra were recorded on Bruker AV III 300 and 400 MHz NMR spectrometers equipped with BBO or BBFO probeheads. Assignment of proton and carbon atoms is based on COSY, NOESY, edited-HSQC and HMBC experiments. ${ }^{1} \mathrm{H}$ and ${ }^{13} \mathrm{C}$ NMR chemical shifts were reported in parts per million (ppm) using residual solvent signal as reference. High-resolution mass spectra were obtained on a Varian MAT 311 or ZabSpec TOF Micromass instrument at CRMPO (Scanmat, UMS 2001). UV-Visible spectra were recorded at room temperature on a VARIAN Cary 5000 spectrophotometer. The UV-Vis emission and excitation spectra measurements were recorded on a FL 920 Edimburgh Instrument equipped with a Hamamatsu R5509-73 photomultiplier for the NIR domain (300-1700 nm) and corrected for the response of the photomultiplier. Quantum yields in solution were calculated relative to quinine sulfate $\left(\mathrm{H}_{2} \mathrm{SO}_{4}, 0.1 \mathrm{M}\right), \phi_{\mathrm{ref}}=0.55$. The absolute quantum yields were measured with a C9920-03 Hamamatsu. phenyldibenzophosphole, ${ }^{1} \mathbf{2}^{2}$ and $\mathbf{4}^{3}$ were synthesized according to published procedure. The FTIR spectra measurements were recorded on a Cary 630 Agilent instrument. Size distributions of the nanoparticles and nanohybrids in solution were determined with DLS using a NanoZetaSizer from Malvern.

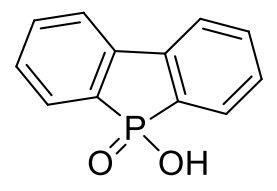

To a solution of dibenzophosphole $(200 \mathrm{mg}, 0.76 \mathrm{mmol})$ in dry THF $(20 \mathrm{~mL})$ is added lithium wire. When the colour turned to black, the remaining lithium wire is removed and the solution is hydrolysed by water and treated by $1 \mathrm{~mL}$ of hydrogen peroxide. The solution is concentrated and the crude mixture is dissolved in DCM and filtered on celite. The crude mixture was extracted with water and then acidification of the aqueous phase led to precipitation of a white solid (20\%).

${ }^{1} \mathrm{H}$ NMR (400 MHz, MeOD) : $\delta 7.46$ (dddd, $J=2 \mathrm{~Hz}, J=4 \mathrm{~Hz}, J=8 \mathrm{~Hz}, J=8 \mathrm{~Hz}, 2 \mathrm{H}$ ) ; 7.63 (dddd, $J=$ $2 \mathrm{~Hz}, J=2 \mathrm{~Hz}, J=8 \mathrm{~Hz}, J=8 \mathrm{~Hz}, 2 \mathrm{H}) ; 7.72(\mathrm{dddd}, J=2 \mathrm{~Hz}, J=2 \mathrm{~Hz}, J=4 \mathrm{~Hz}, J=8 \mathrm{~Hz}, 2 \mathrm{H}) ; 7.91$ (dddd, $J=2 \mathrm{~Hz}, J=4 \mathrm{~Hz}, J=4 \mathrm{~Hz}, J=8 \mathrm{~Hz}, 2 \mathrm{H}) \cdot{ }^{13} \mathrm{C}$ NMR $(100 \mathrm{MHz}, \mathrm{MeOD}): 122.7(\mathrm{~d}, J(\mathrm{P}, \mathrm{C})=12$ $\mathrm{Hz}) ; 129.2(\mathrm{~d}, J(\mathrm{P}, \mathrm{C})=9 \mathrm{~Hz}) ; 130.8(\mathrm{~d}, J(\mathrm{P}, \mathrm{C})=11 \mathrm{~Hz}) ; 131.8(\mathrm{~d}, J(\mathrm{P}, \mathrm{C})=137 \mathrm{~Hz}) ; 135.0(\mathrm{~d}, J(\mathrm{P}, \mathrm{C})$ $=2 \mathrm{~Hz}) ; 141.6(\mathrm{~d}, J(\mathrm{P}, \mathrm{C})=29 \mathrm{~Hz}) .{ }^{31} \mathrm{P}$ NMR $(162 \mathrm{MHz}): \delta+40.0$. HRMS (EI) : 215.0265 calc. For $\mathrm{C}_{12} \mathrm{H}_{8} \mathrm{O}_{2} \mathrm{P}: 215.0267$.

\footnotetext{
${ }^{1}$ A. Oukhrib, L. Bonnafoux, A. Panossian, S. Waifang, D. H. Nguyen, M. Urrutigoity, F. Colobert, M. Gouygou, F. R. Leroux, Tetrahedron 2014, 70, 1431.

${ }^{2}$ Rabah, A. Escola, O. Jeannin, P.-A. Bouit, M. Hissler, F. Camerel, ChemPlusChem 2020, 85, 79

${ }^{3}$ R. Szücs, F. Riobé, A. Escande, D. Joly, P.-A. Bouit, L. Nyulászi, M. Hissler, Pure Appl. Chem. 2017, 2017, 88, 341.
} 


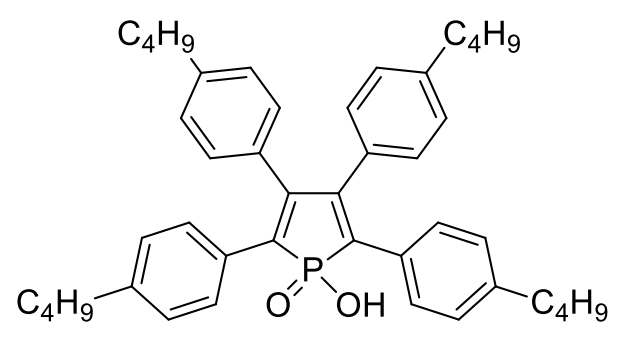

3. 1,2-bis(4-butylphenyl)ethyne (500 mg, $1.7 \mathrm{mmol}, 2$ eq.) $\mathrm{ZrCp}_{2} \mathrm{Cl}_{2}$ (264 mg, 1.05 eq.) are dissolved in THF (20 mL). BuLi (0,76 mL, 2.2 eq.) is added at $-78^{\circ} \mathrm{C}$ and stirred at RT overnight. $\mathrm{CuI}(344 \mathrm{mg}, 2,1 \mathrm{eq})$ is then added at $0^{\circ} \mathrm{C}$ and the solution is stirred $1 \mathrm{~h} . \mathrm{PCl}_{3}(0.3 \mathrm{~mL}, 4$ eq. $)$ solvent is then evaporated and the crude is dissolved in THF. the reaction is hydrolyzed with water and treated by $1 \mathrm{~mL}$ of hydrogen peroxide. The solution is concentrated and the crude is purified by column

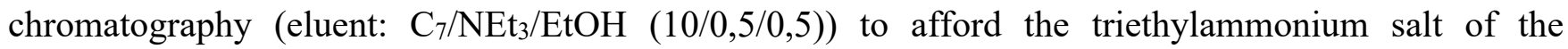
compound. The compound is dissolved in DCM, acidified with 10\% HBF4 aqueous solution, washed with water. The solvent is evaporated to afford the compound as a yellow solid $(\mathrm{r}=45 \%)$.

${ }^{1} \mathrm{H}$ NMR (400 MHz, $\left.\mathrm{CD}_{2} \mathrm{Cl}_{2}\right): \delta 0.88(\mathrm{t}, J=7.3 \mathrm{~Hz}, 6 \mathrm{H}) ; 0.92(\mathrm{t}, J=6.9 \mathrm{~Hz}, 6 \mathrm{H}) ; 1.18-1.40(\mathrm{~m}, 8 \mathrm{H})$; $1.45-1.65(\mathrm{~m}, 8 \mathrm{H}) ; 2.53(\mathrm{t}, J=7.7 \mathrm{~Hz}, 8 \mathrm{H}) ; 6.77(\mathrm{~d}, J=7.8 \mathrm{~Hz}, 4 \mathrm{H}) ; 6.87(\mathrm{~d}, J=8.0 \mathrm{~Hz}, 4 \mathrm{H}) ; 6.92$ $(\mathrm{d}, J=7.9 \mathrm{~Hz}, 4 \mathrm{H}) ; 7.26(\mathrm{~d}, J=7.8 \mathrm{~Hz}, 4 \mathrm{H}) .{ }^{13} \mathrm{C} \mathrm{NMR}\left(100 \mathrm{MHz}, \mathrm{CD}_{2} \mathrm{Cl}_{2}\right): \delta 14.2 ; 22.6 ; 23.1 ; 33.8$; $33.9 ; 35.8 ; 36.0 ; 128.0(\mathrm{~d}, J(\mathrm{C}, \mathrm{P})=120 \mathrm{~Hz}, C) ; 128.5(\mathrm{~s}, \mathrm{CH}) ; 128.8(\mathrm{~s}, \mathrm{CH}) ; 129.6(\mathrm{~d}, J=6.0 \mathrm{~Hz}$, $\mathrm{CH}) ; 129.9(\mathrm{~s}, \mathrm{CH}) ; 130.3(\mathrm{~d}, J=9.7 \mathrm{~Hz}, \mathrm{C}) ; 133.0(\mathrm{~d}, J=20.9 \mathrm{~Hz}, \mathrm{C}) ; 143.1(\mathrm{~s}, \mathrm{C})$; 143.2 (s, C) 149.4 $(\mathrm{d}, J=35 \mathrm{~Hz}, \mathrm{C}) .{ }^{31} \mathrm{P}$ NMR $\left(81 \mathrm{MHz} ; \mathrm{CD}_{2} \mathrm{Cl}_{2}\right):+55.0 \mathrm{HRMS}$ (ESI) : 643.3707: calc. For $\mathrm{C}_{44} \mathrm{H}_{52} \mathrm{O}_{2} \mathrm{P}$ : 643.3710. Elem. Anal calcd for $\mathrm{C}_{44} \mathrm{H}_{52} \mathrm{O}_{2} \mathrm{P}: \mathrm{C}, 81.95$, H 8.28. Found C, 81.78, H, 8.20. 


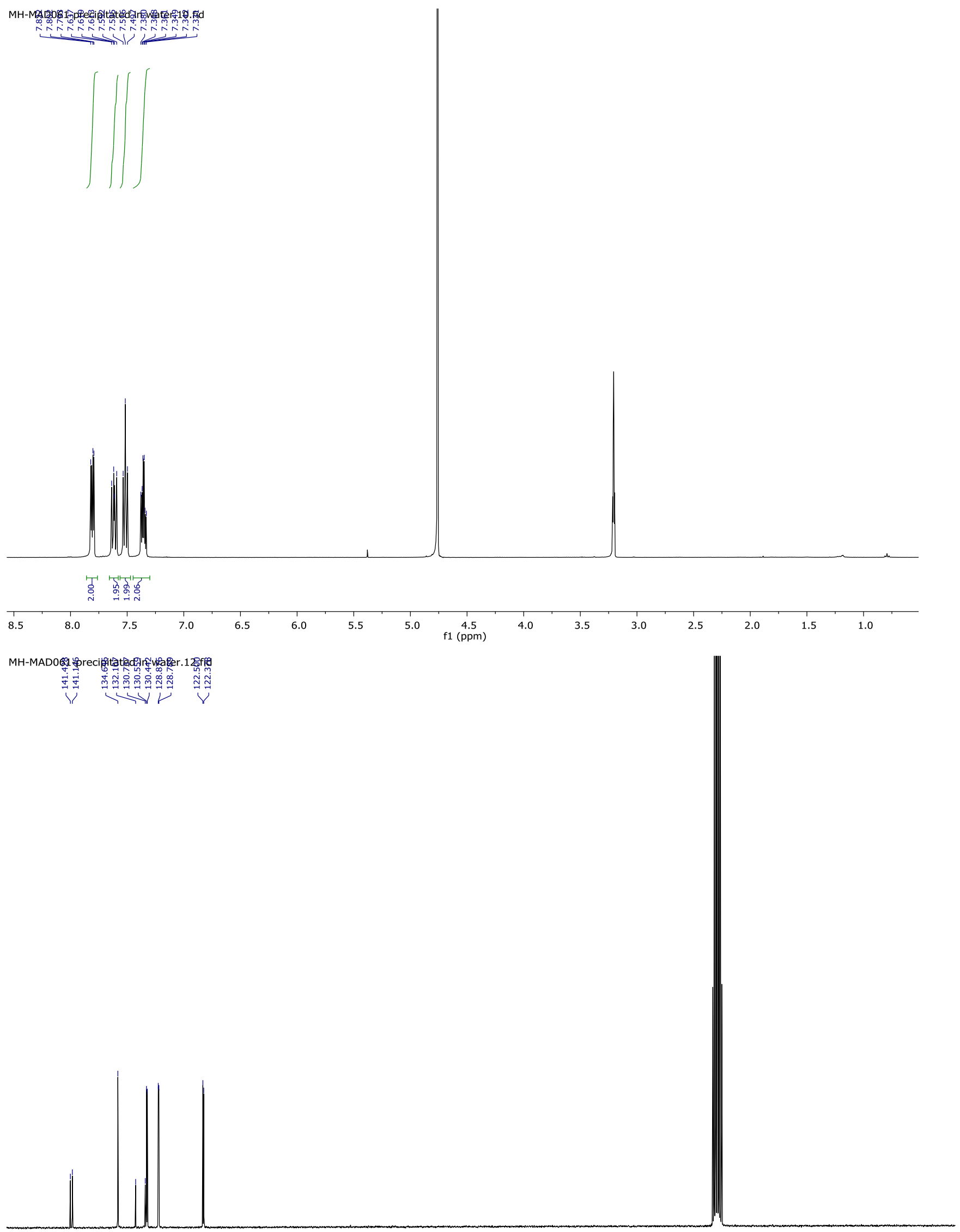

$\begin{array}{llllllllllllllllllllllllllllllllll}50 & 145 & 140 & 135 & 130 & 125 & 120 & 115 & 110 & 105 & 100 & 95 & 90 & 85 & 80 & 75 & 70 & 65 & 60 & 55 & 50 & 45 & 40 & 35 & 30 & 25 & 20 & \end{array}$ 


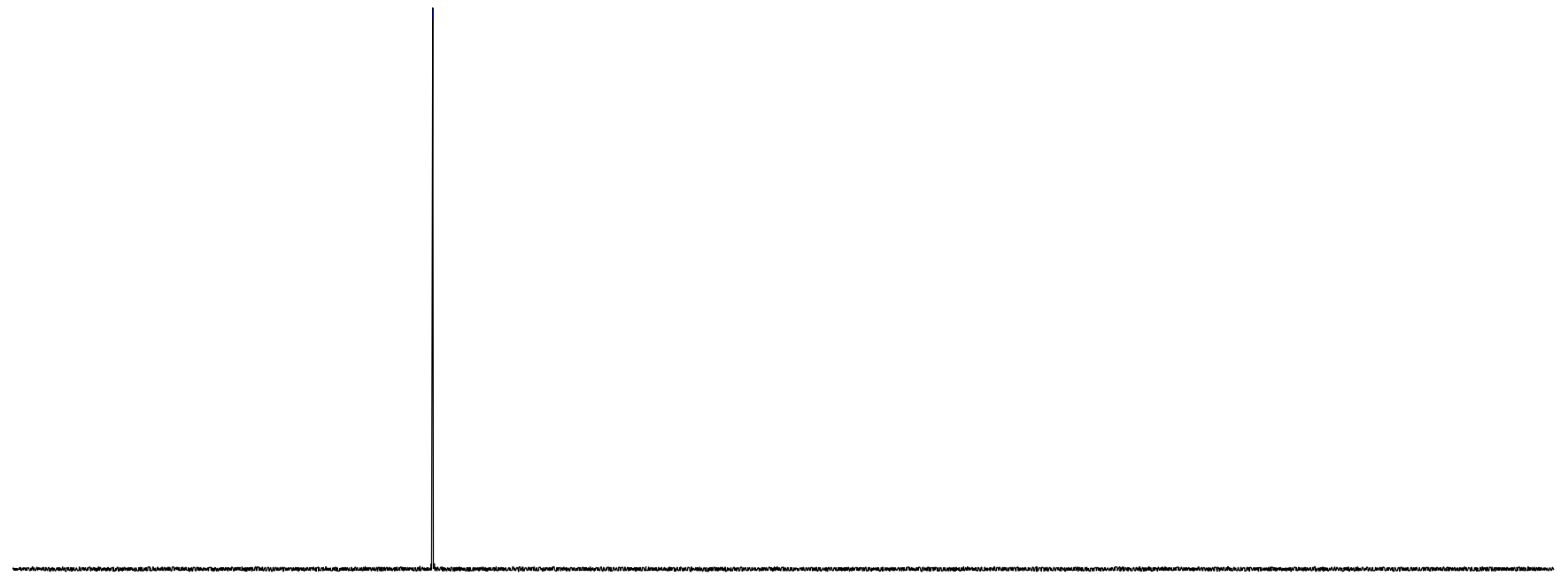

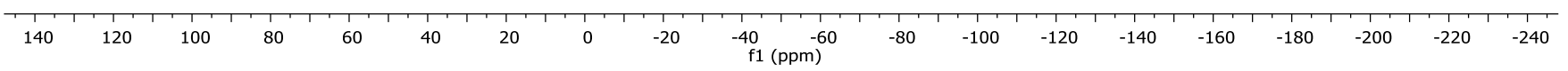

Figure $\mathrm{S} 1:{ }^{1} \mathrm{H},{ }^{13} \mathrm{C}$ and ${ }^{31} \mathrm{P}$ NMR spectrum of 1.

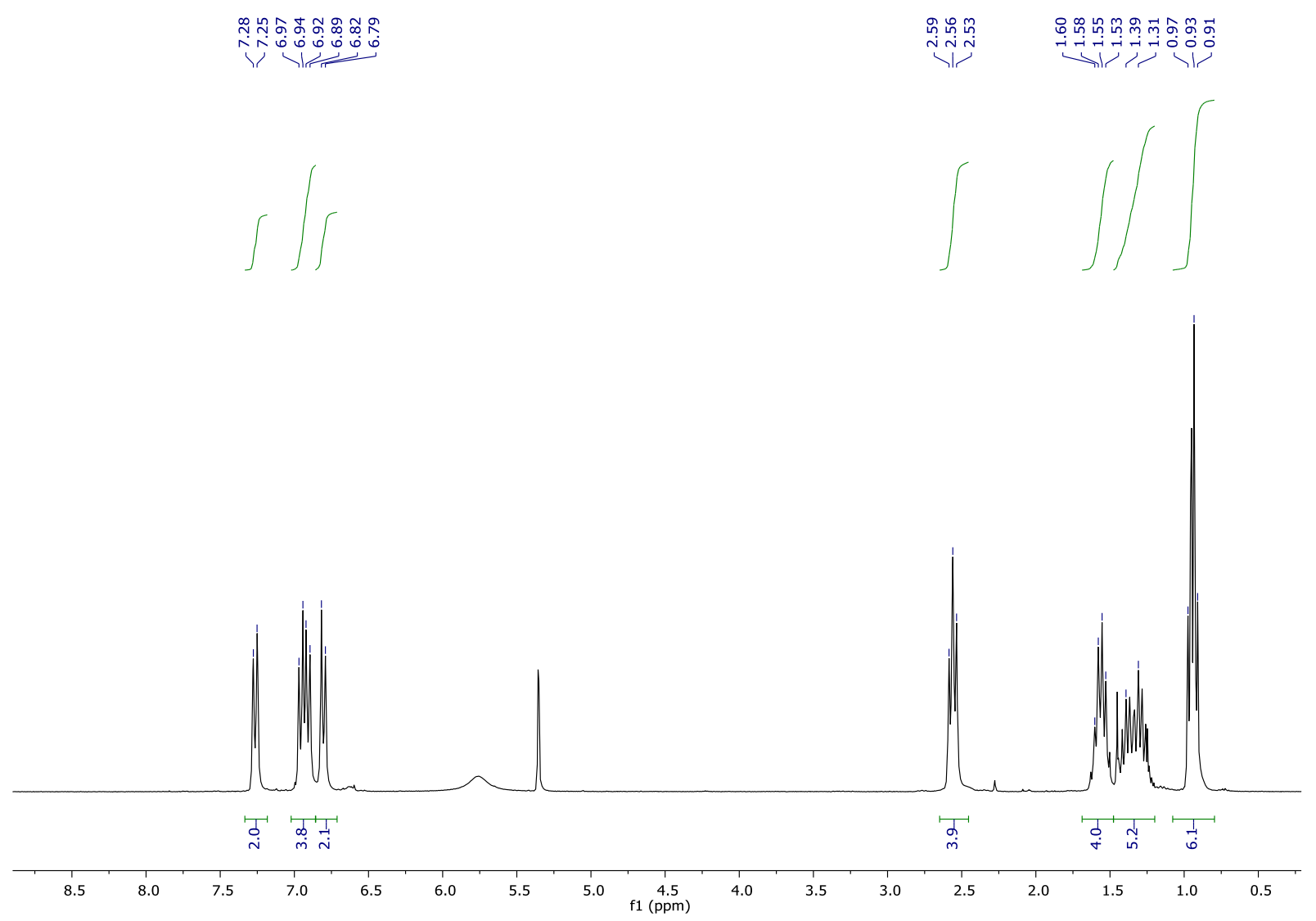



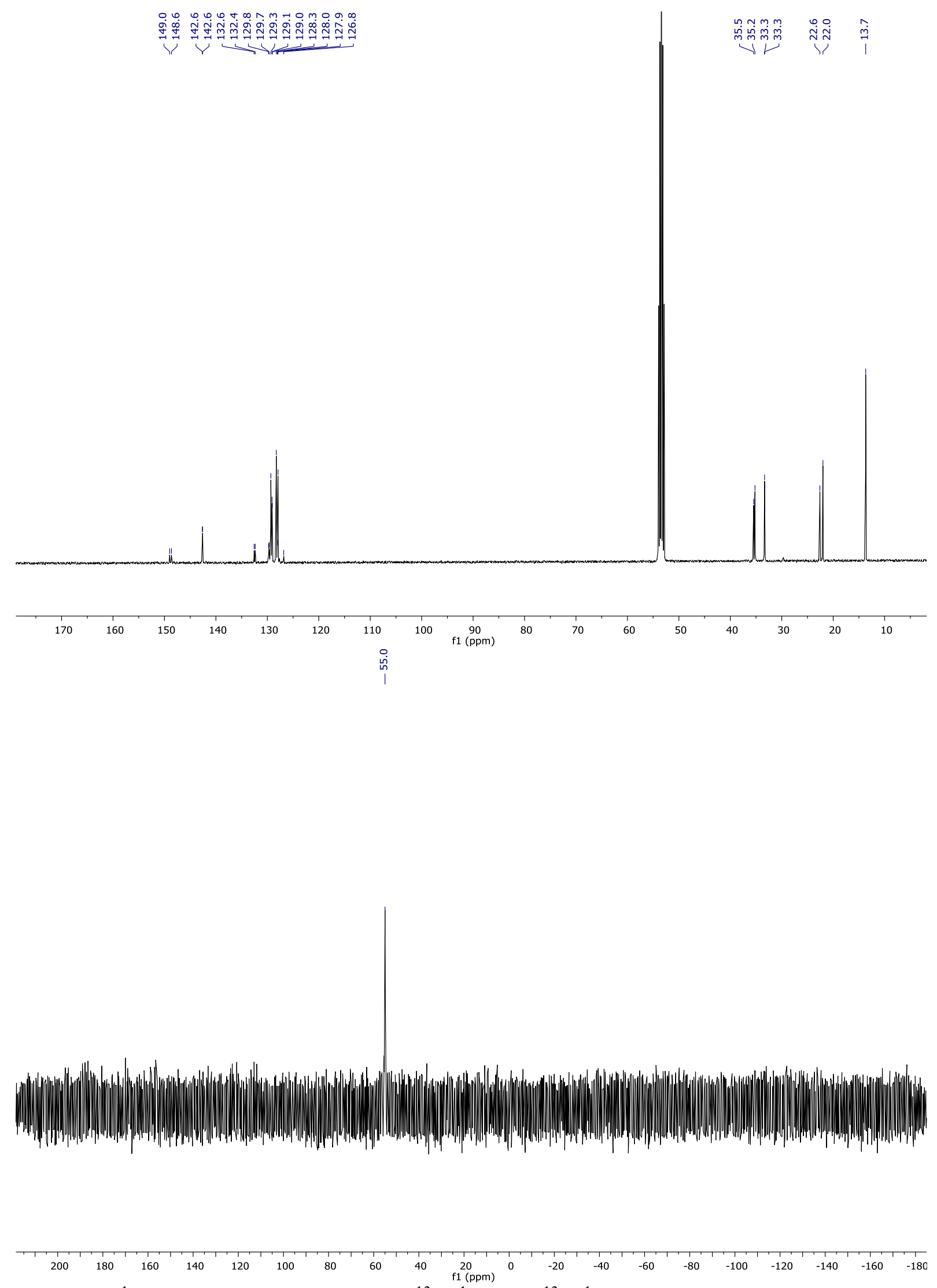

Figure S2: ${ }^{1} \mathrm{H}$ NMR $\left(400 \mathrm{MHz}, \mathrm{CD}_{2} \mathrm{Cl}_{2}\right),{ }^{13} \mathrm{C}\left\{{ }^{1} \mathrm{H}\right\}$ and ${ }^{13} \mathrm{P}\left\{{ }^{1} \mathrm{H}\right\}$ NMR spectra of 3 


\section{$\underline{\text { X-ray Crystallographic Study: }}$}

Single crystals suitable for X-Ray crystal analysis were obtained by slow diffusion of vapors of pentane into a dichloromethane solution of the derivatives at rt. Single crystal data collection were performed at $150 \mathrm{~K}$ with an D8 VENTURE Bruker AXS diffractometer equipped with a (CMOS) PHOTON 100 detector with Mo- $K \alpha$ radiation $(\lambda=0.71073 \AA)$. The structure was solved by dual-space algorithm using the SHELXT program ${ }^{4}$, and then refined with full-matrix least-squares methods based on $F^{2}$ (SHELXL). ${ }^{5}$ All non-hydrogen atoms were refined with anisotropic atomic displacement parameters. $\mathrm{H}$ atoms were finally included in their calculated positions and treated as riding on their parent atom with constrained thermal parameters.

Table S1: Crystal data and structure refinement

\section{Compound}

CCDC

Formula

MW

$\mathrm{a}(\AA)$

$\mathrm{b}(\AA)$

c $(\AA)$

$\alpha\left(^{\circ}\right)$

$\beta\left(^{\circ}\right)$

$\gamma\left({ }^{\circ}\right)$

$\mathrm{V}\left(\AA^{3}\right)$

$\mathrm{Z}$

$D c\left(\mathrm{~g} . \mathrm{cm}^{-3}\right)$

Crystal system

Space group

$\mathrm{T}(\mathrm{K})$

Wavelength Mo-K $\alpha(\AA)$

$\mu\left(\mathrm{mm}^{-1}\right)$

$F(000)$

$\theta$ limit $\left(^{\circ}\right)$

Index ranges $h k l$

Reflections collected

Independant reflections

Reflections $[l>2 \sigma(l)]$

Data / restraints / parameters
3

1919421

$\mathrm{C}_{44} \mathrm{H}_{53} \mathrm{O}_{2} \mathrm{P}$

644.83

$9.2698(8)$

$14.1158(13)$

$16.1772(15)$

68.005(3)

77.851(3)

78.264(4)

1900.6(3)

2

1.127

triclinic

$P-1$

150

$0.71073 \AA$

0.107

696

3.005 to 27.484

$-11 \leq \mathrm{h} \geq 12$

$-18 \leq \mathrm{k} \geq 18$

$-20 \leq 1 \geq 20$

35337

8681

6880

${ }^{4}$ G. M. Sheldrick, Acta Cryst., 2015, A71, 3-8

${ }^{5} \mathrm{G}$. M. Sheldrick, Acta Cryst., 2015, C71, 3-8 
Goodness-of-fit on $F^{2}$

Final $R$ indices $[1>2 \sigma(l)]$

$\mathrm{R}$ indices (all data)

Largest diff peak and hole (e $\left.\AA^{-3}\right)$
1.012

$\mathrm{R} 1=0.0488$

$w R 2=0.1177$

$R 1=0.0671$

$w R 2=0.1291$

0.825 and -0.576

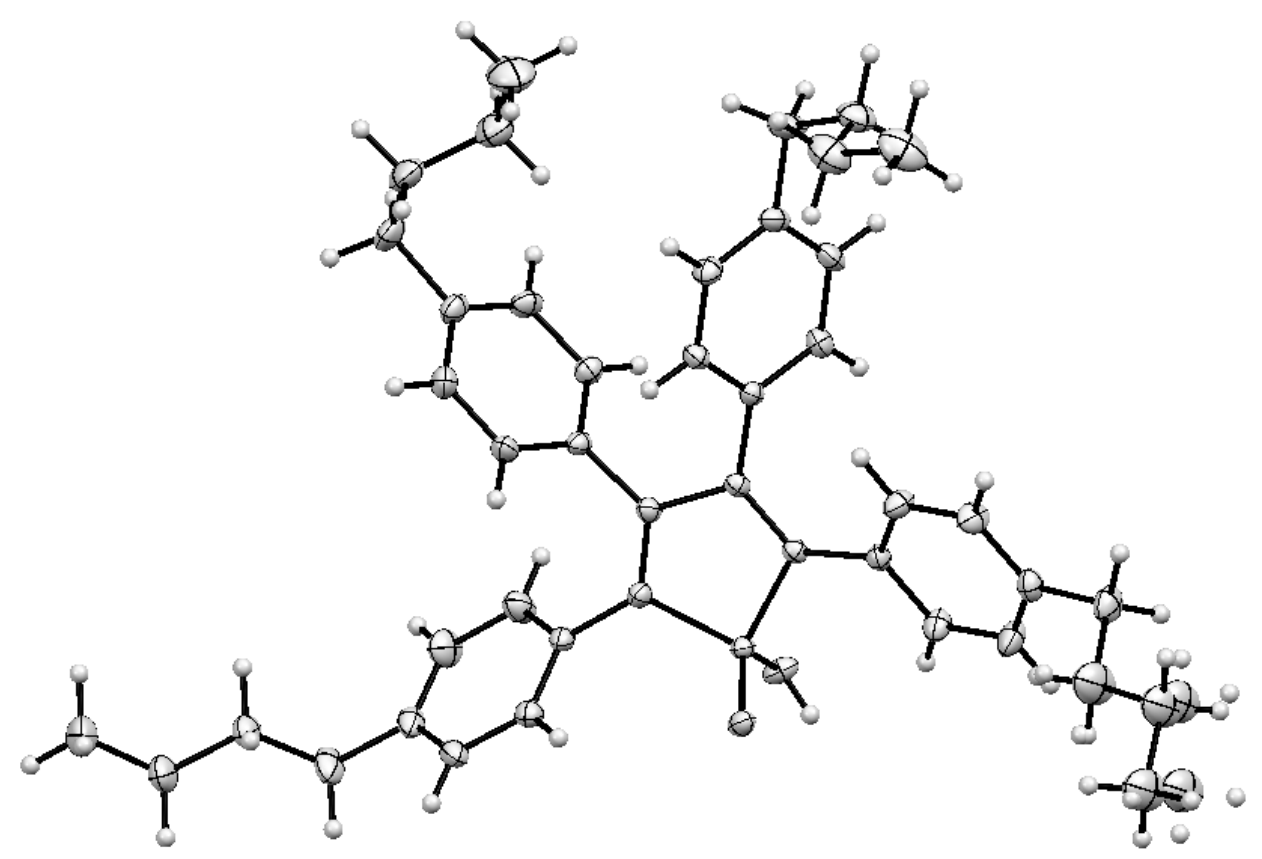

Figure S3: ORTEP representation of 3 with 50\% probability ellipsoids 


\section{Theoretical Methods:}

We generated models of tetraphenylhydroxyoxophosphole/ZnO hybrid interface by atomistic simulations combining classical molecular dynamics (MD) for pre-optimization and Density Functional Theory (DFT) for optimization and electronic characterization. Concerning DFT, the PBE exchange correlation functional was adopted for the geometrical optimization while the DFT+U correction was applied to properly characterize the electronic structure of the final hybrid organic/inorganic structure. We made use of the planewave pseudopotential approach as implemented in Quantum ESPRESSO (Quantum opEnSource Package for Research in Electronic Structure, Simulation, and Optimisation).[1,2] Ultrasoftpseudo potentials were used for all atomic species. Plane wave basis set with 35 Ry cutoff for kinetic energy, and 280 Ry for the electronic density and potential. The threshold for self-consistent convergence was chosen as small as $10^{-8}$.

DFT calculations of electronic properties of $\mathrm{ZnO}$ systems are affected by well-known inaccuracies: underestimation of the electronic gap and a wrong hybridization between the $3 \mathrm{~d} \mathrm{Zn}$ states with $2 \mathrm{p} \mathrm{O}$ states; to correct such inaccuracies and achieve a better characterization of the electronic properties it is necessary to apply beyond-DFT methods. For example, the VPSIC method (virtual pseudo-selfinteraction correction) on the clean $\mathrm{ZnO}$ slab yields an electronic gap of the zinc oxide close to the experimental value with the $3 \mathrm{~d} \mathrm{Zn}$ bands forming a distinct manyfold well separated from the $2 \mathrm{p} O$ valence bands.[3] Here, for calculating the electronic structure of the hybrid systems, we made use of the DFT + U method by selecting the onsite Hubbard correction to reproduce the same VPSIC offset between the $\mathrm{Zn} 3 \mathrm{~d}$ on $\mathrm{O} 2 \mathrm{p}$ bands. By combining the DFT and DFT $+\mathrm{U}$ methods it was possible to keep low the computational cost and to get a reliable description of the band alignment of the molecule/surface in its minimum energy configuration with a precise estimation of the binding mechanism. Concerning the kpoint mesh, we made use of the zone center point $\Gamma$ for the relaxation of the grafted systems and of a $2 x$ $2 \times 1$ k-point Monkhorst-Pack mesh for the electronic structure on the optimized geometry.

The initial atomistic configurations were obtained by placing the molecule vertically aligned at different positions on top of a relaxed $\mathrm{ZnO}$ (10-10) non-polar surface (that is also known to be the most stable). Periodic boundary conditions were applied along all Cartesian directions. An empty region of $2.0 \mathrm{~nm}$ was introduced in the vertical $z$ direction to model the surface. This approach is standard to study surfaces by using plane-waves methods and periodic simulation boxes. Classical molecular dynamics were used to pre-relax the interface at finite low temperature and to identify realistic bound initial configurations. To this aim we made use of empirical force-fields (i.e. GAFF plus LJ-Coulomb interactions) previously applied by the authors to the study of organic/ZnO interfaces.[4-5] The obtained systems (containing 180 atoms) were further relaxed by DFT. Ionic optimization made use of the BFGS optimization algorithm. 
We considered both the neutral molecule with full POOH group as well as the deprotonated configuration with POO- termination and the proton $\mathrm{H}+$ either put on the surface or removed from the box. We verified that the neutral molecule tends to spontaneously release the $\mathrm{H}+$ proton during optimization on the surface. Our results indicate that the system converges to a bi-dentate anchoring conformation (see Figure S4A) that represents the lowest energy bound state for the molecule on the surface. The energy profile calculated by classical MD during the bi-dentate anchoring of the molecule is reported in Figure S4B indicating a strong binding of about $2.8 \mathrm{eV}$ per $\mathrm{O}(\mathrm{mol})-\mathrm{Zn}$ (surface) link.

Charge energy difference were obtained by taking the difference between the electronic density of the molecule in the bound system and in the case of an isolated molecule in the same simulation box. The marker atom method was used to align electronic structure and density of states obtained in separate calculations. The position of a core level electron of an atom far apart from the binding site is used to align the density of state of the $\mathrm{ZnO}$ free surface, the molecule and the bound system. The position of the Fermi level is obtained by applying the Fermi-Dirac occupation to the calculated density of states. The electronic structure of the grafted molecule shows a downshift of the molecular levels and a weak charge displacement only visible at the O-Zn bond.

Electronic material (including XYZ files of relevant configurations) is available upon request at https://www.dsf.unica.it/ mattoni

\section{References}

[1] P. Giannozzi et al., J.Phys.:Condens.Matter 21, 395502 (2009) http://dx.doi.org/10.1088/0953$8984 / 21 / 39 / 395502$

[2] P. Giannozzi et al., J.Phys.:Condens.Matter 29, 465901 (2017)

http://iopscience.iop.org/article/10.1088/1361-648X/aa8f79

[3] A. Filippetti et al., Phys. Rev. B., 84, 195127 (2011)

https://link.aps.org/doi/10.1103/PhysRevB.84.195127

[4] C. Caddeo et al. J. Phys. Chem. C 2011, 115, 34, 16833-16837

https://doi.org/10.1021/jp204238m

[5] E. Javon et al. ACS Nano 2015, 9, 4, 3685-3694

https://doi.org/10.1021/acsnano.5b00809 

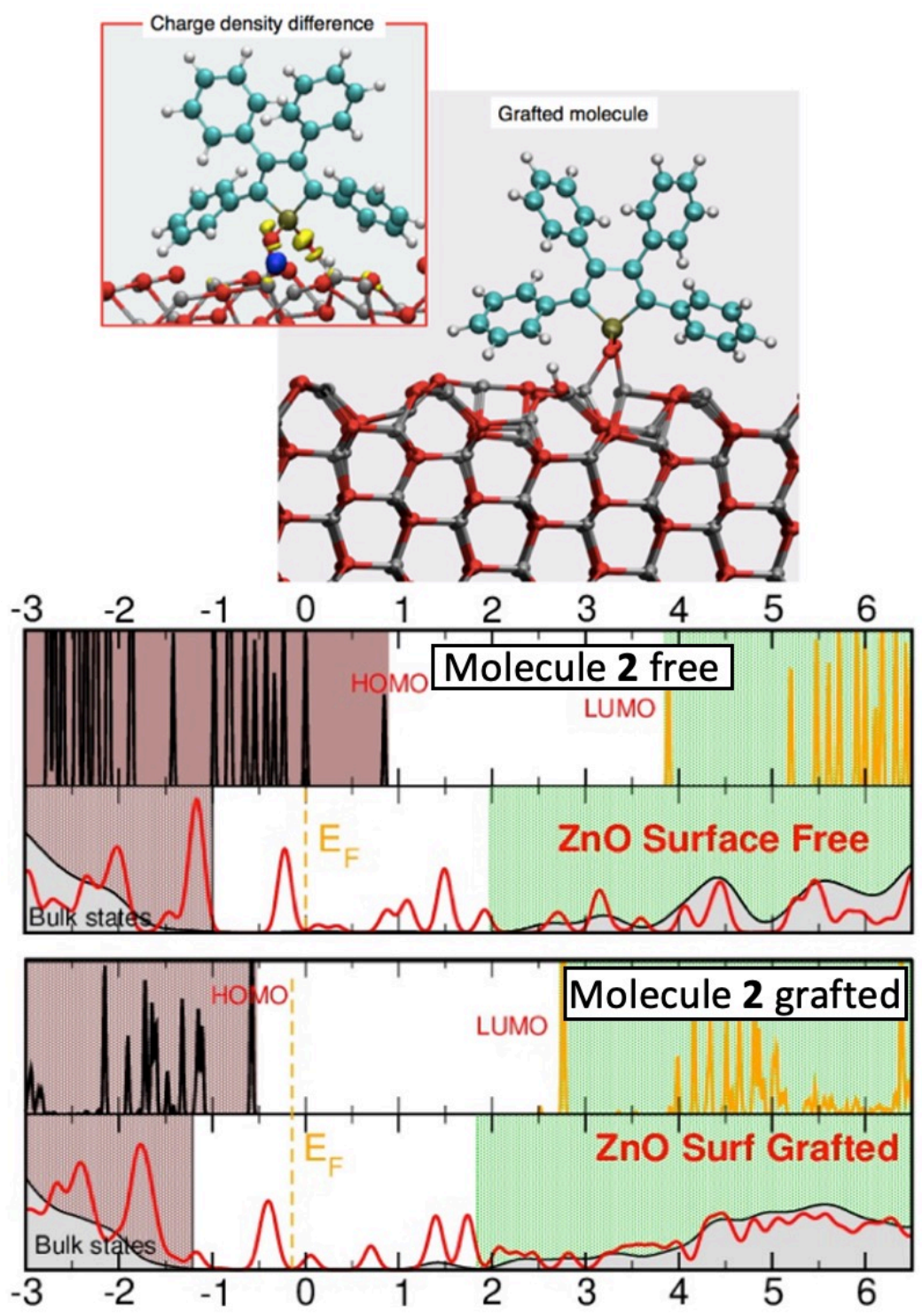

Figure S4A: DFT calculation of the interactions of $\mathbf{2}$ and the $\mathrm{ZnO}$ surface to form $\mathrm{ZnO}: \mathbf{2}$ nanohybrids.

Top: Grafted molecule on the surface (right) and charge energy difference upon grafting (inset).

Bottom: Band alignment of the molecular levels with respect to the surface for the case of separated components (upper panels) and after grafting (lower panels). Green (brown) shadowed regions indicate the empty (full) electronic levels of the molecule and the surface. Using the marker atom method, the electronic levels have been aligned. It is found a downshift of the molecular levels upon grafting. 

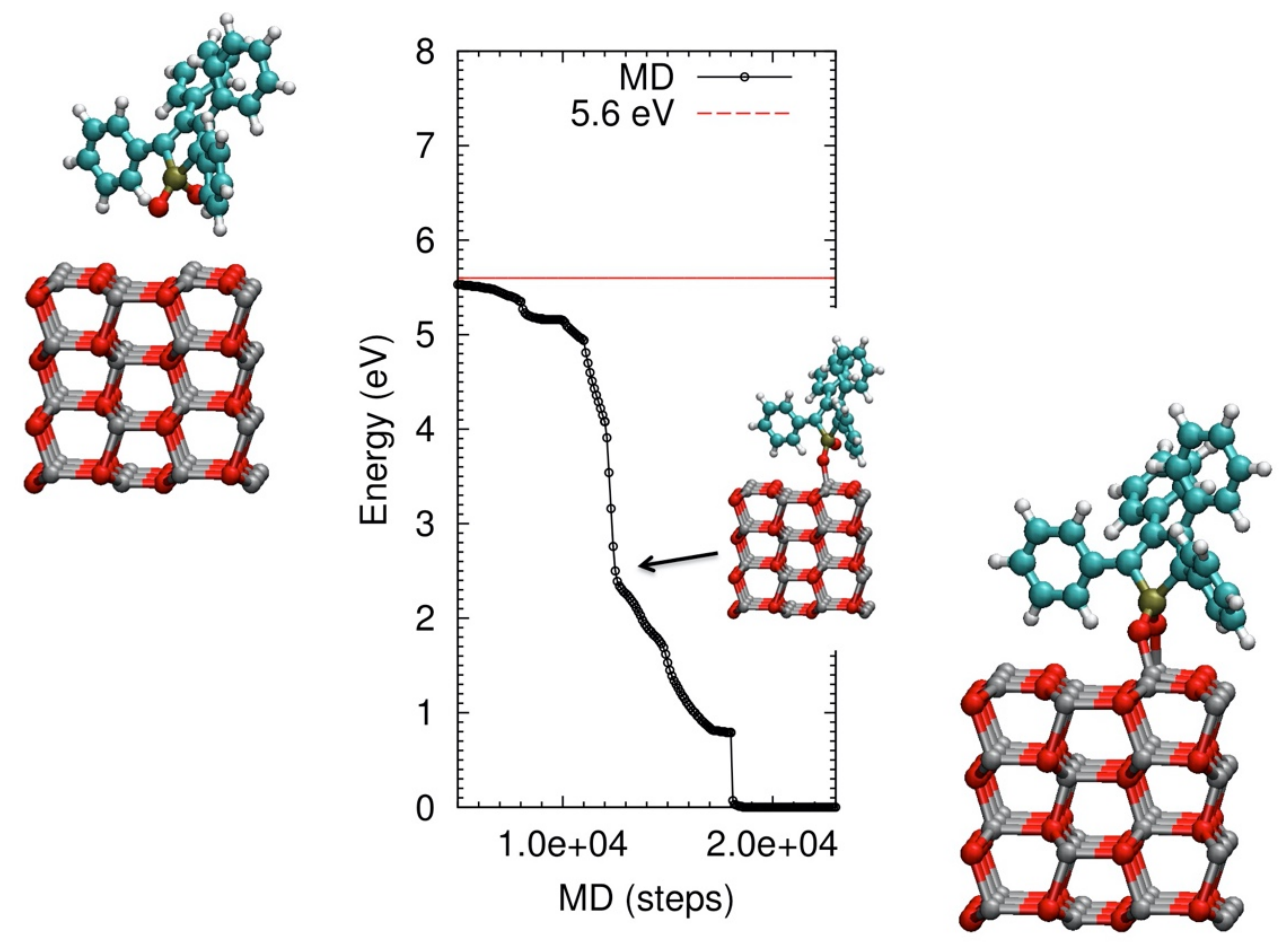

Figure S4B: Energy profile calculated by MD during the anchoring of the de-protonated tetraphenylhydroxyoxoposphole in the bi-dentate configuration on top of the (10-10) ZnO surface. The bidentate bound state occurs through the subsequent formation of two $\mathrm{O}$ (mol)-Zn(surface) links each providing a binding energy of about 2.5-2.8 eV (according to the classical force field). 


\section{$\underline{\text { XPS data - Methods: }}$}

The x-ray photoelectron spectroscopy (XPS) experiments were done in an UHV surface analysis system, consisting of an entry chamber (base pressure $\approx 1 \times 10^{-7} \mathrm{mbar}$ ), a preparation chamber $\left(\approx 1 \times 10^{-9} \mathrm{mbar}\right.$ ), and an analysis chamber $\left(\approx 5 \times 10^{-10} \mathrm{mbar}\right)$. The XPS (monochromatized Al Ka hv $=1486.6 \mathrm{eV}$ ) spectra were recorded with a Scienta-200 hemispherical analyzer and calibrated by referencing to the Fermi level edge and $\mathrm{Au} 4 \mathrm{f} 7 / 2$ peak position of an $\mathrm{Ar}^{+}$ion sputter-cleaned gold foil. Thin films of compound 2 and ZnO:2 were spin-coated onto gold substrates and transferred from the fume hood into the entry chamber for subsequent measurements.

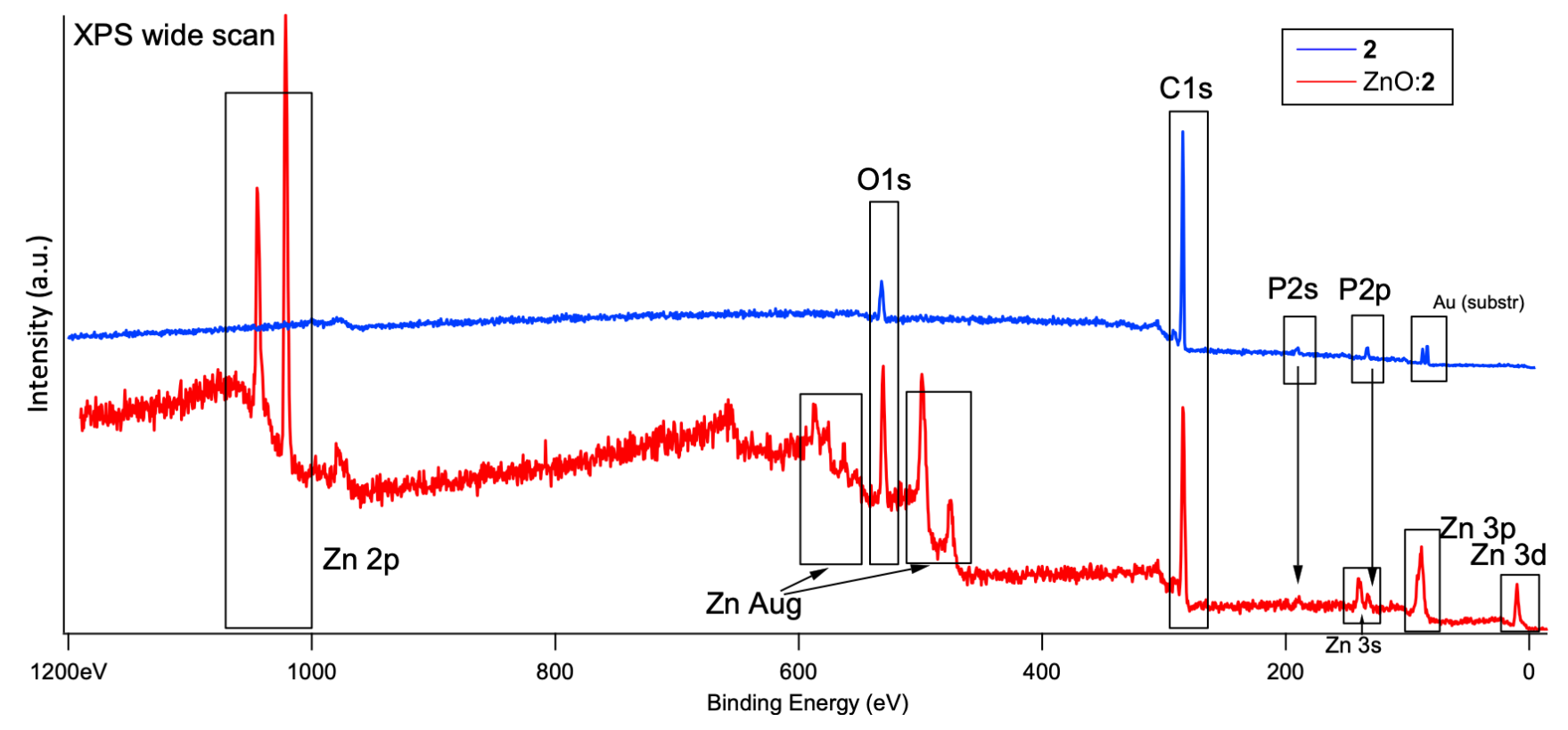

Figure S5: XPS wide scan of compounds 2 and $\mathrm{ZnO:2.}$ 


\begin{tabular}{llllll}
\hline \multirow{2}{*}{ Compound } & $\begin{array}{l}\lambda_{\text {abs }}^{\text {liq[a] }} \\
{[\mathrm{nm}]}\end{array}$ & $\begin{array}{l}\lambda_{\mathrm{em}}^{\text {liq[a] }} \\
{[\mathrm{nm}]}\end{array}$ & $\begin{array}{l}\phi_{\mathrm{liq}}^{[\mathrm{a}, \mathrm{b}]} \\
{[\%]}\end{array}$ & $\begin{array}{l}\lambda_{\mathrm{em}}{ }^{\text {solid[d] }} \phi_{\text {powder }}{ }^{[\mathrm{d}, \mathrm{e}]} \\
{[\mathrm{nm}]}\end{array}$ & {$[\%]$} \\
\hline $\mathbf{1}$ & 323 & 363 & $26^{[\mathrm{c}]}$ & 394 & - \\
$\mathbf{2}$ & 378 & 513 & 0.1 & 534 & 14 \\
$\mathbf{3}$ & 380 & 545 & 0.2 & 542 & 15 \\
$\mathbf{4}$ & 400 & 524 & 0.5 & 532 & 46
\end{tabular}

[a] measured in DCM solution $\left(\mathrm{c}=10^{-5} \mathrm{M}\right)$. [b] Measured relative to quinine sulfate $\left(\mathrm{H}_{2} \mathrm{SO}_{4}, 0.1 \mathrm{M}\right)$, $\phi_{\text {ref }}=0.54$. [c] Measured relative to anthracene in ethanol, $\phi_{\text {ref }}=0.27$ [d] Measured in powder. [e] absolute quantum yield measured in Hamamatsu integration sphere

Table S2: Photophysical data of ligands
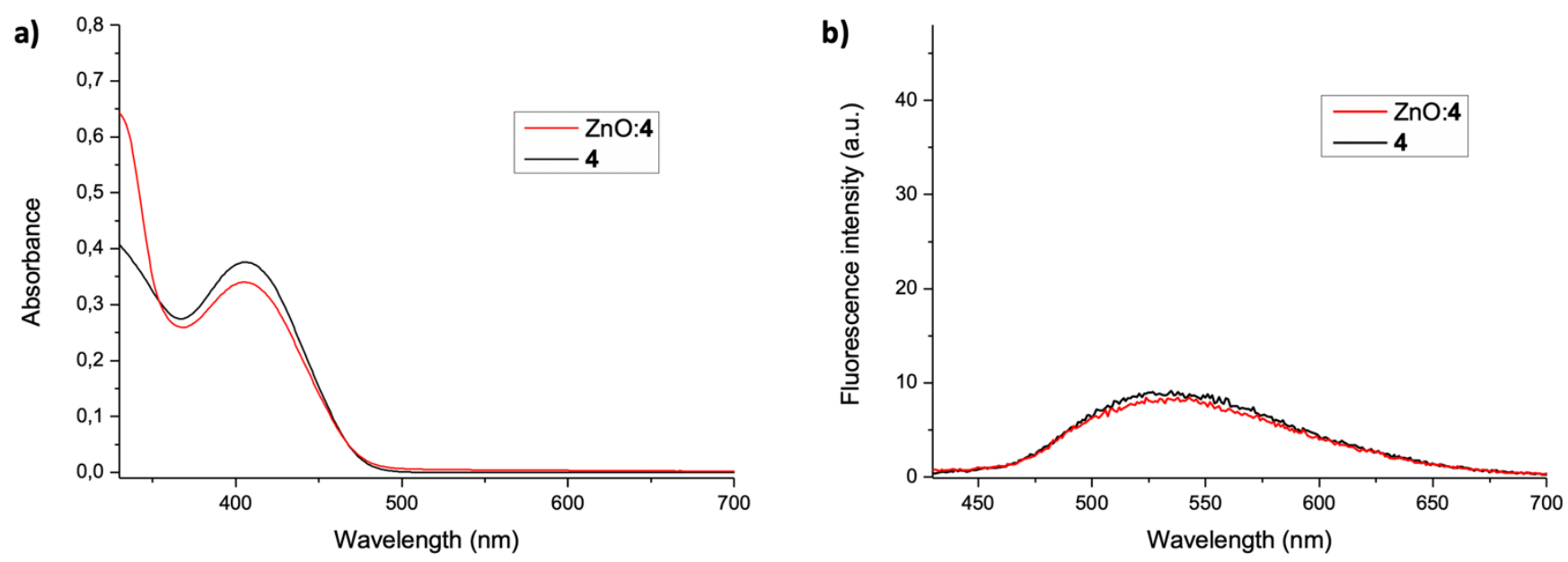

Figure S6: a) absorption and b) fluorescence spectra at $\lambda_{\mathrm{ex}}=400 \mathrm{~nm}$ of the non-graftable molecule 4 (black curves) and the nanohybrids $\mathrm{ZnO} 4$ (red curves). 

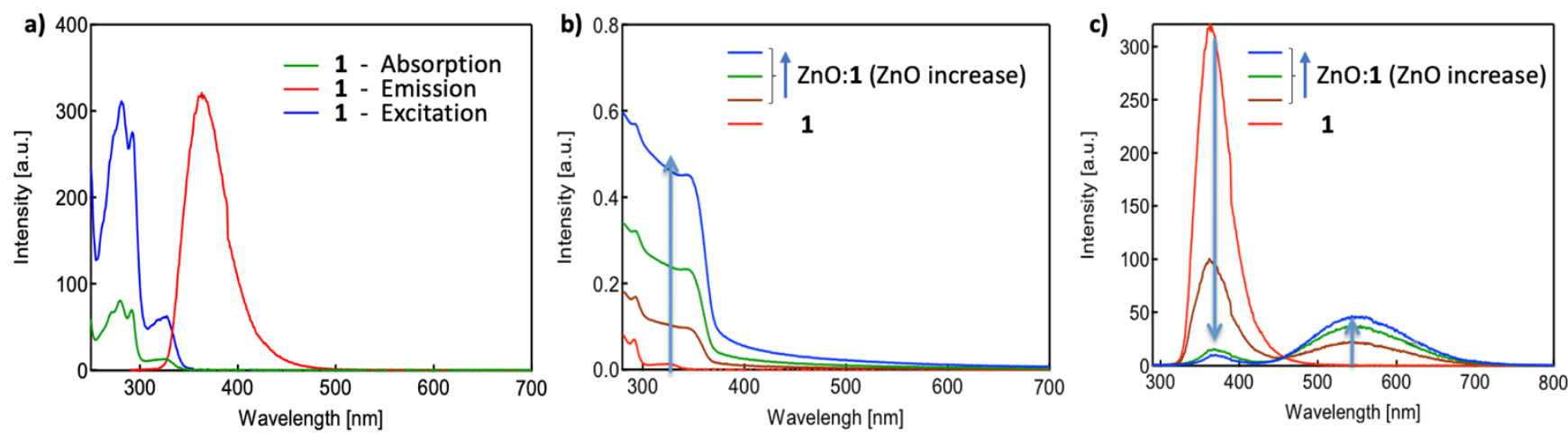

Figure S7: a) Absorption spectrum (in green), emission spectrum at $\lambda_{\mathrm{ex}}=280 \mathrm{~nm}$ (in red) and excitation spectrum measured at $\lambda_{\mathrm{m}}=360 \mathrm{~nm}$ (in blue) for molecule 1 in solution.

b) Absorption spectra of nanohybrids $\mathrm{ZnO}: 1$ when increasing the $\mathrm{ZnO}$ concentration.

c) Fluorescence spectra at $\lambda_{\mathrm{ex}}=280 \mathrm{~nm}$ of the molecule $\mathbf{1}$ and the nanohybrids $\mathrm{ZnO}: \mathbf{1}$ when increasing the $\mathrm{ZnO}$ concentration. Increase of $\mathrm{ZnO}$ in the solution increases the amount of grafted $\mathbf{1}$ but the emission of $\mathbf{1}$ is quenched due to the intermolecular interaction (ACQ effect). 


\section{Transmission Electron Microscopy - Methods:}

The nanohybrids were characterized by HR-TEM (JEOL 3010, acceleration voltage of $300 \mathrm{kV}$ ). The samples were prepared by drop-casting a diluted solution of nanohybrids in chloroform onto a carboncoated copper grid.
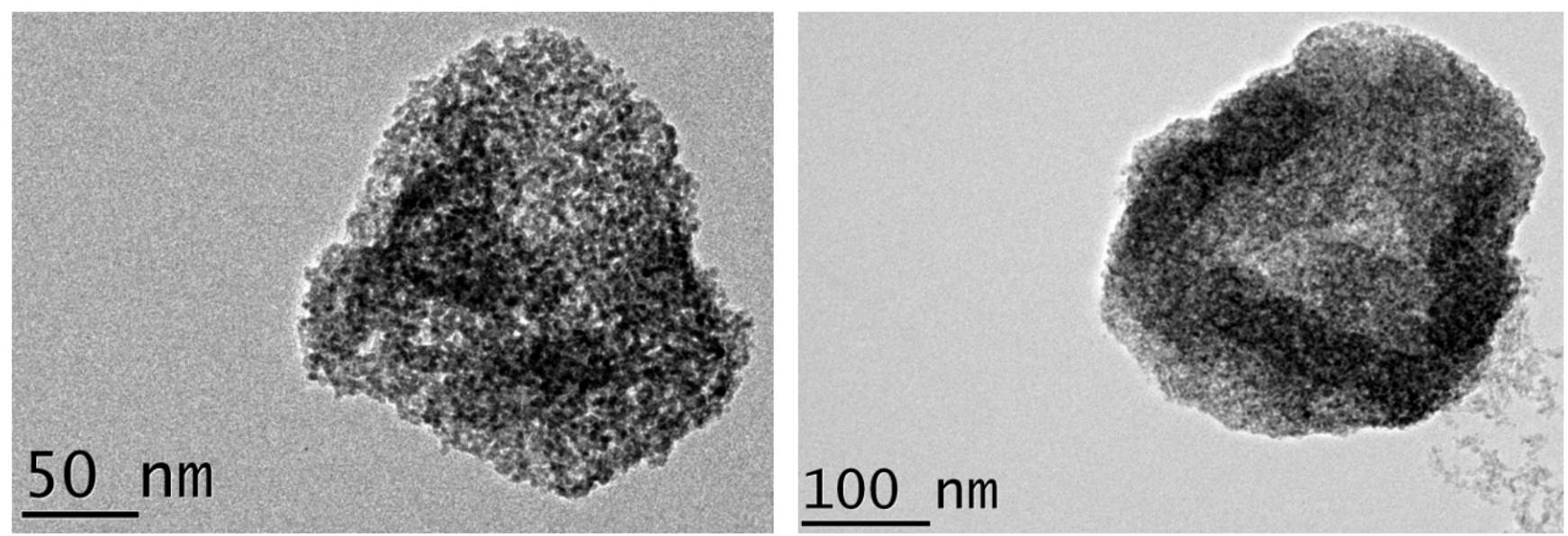

Figure S8: TEM image of nanohybrid aggregates obtained from $\mathrm{ZnO} 22$ solution. 


\section{LED fabrication - Methods:}

The OLED devices were fabricated onto indium tin oxide (ITO) glass substrates purchased from Xin Yang Technology (90 nm thick, sheet resistance of $15 \Omega / \square$ ). Prior to organic layer deposition, the ITO substrates were cleaned by sonication in a detergent solution, rinsed twice in de-ionized water and then in isopropanol solution and finally treated with UV-ozone during 15 minutes. The OLEDs stack is: Glass / ITO/ CuPc/ HTL/ ZnO:3/ mCP/ Alq3/ LiF/ Al with copper (II) phthalocyanine (CuPc, Aldrich), $10 \mathrm{~nm}$ thick film as hole injection layer and $N, N^{\prime}$-dicarbazolyl-3,5-benzene (mCP, Aldrich) $10 \mathrm{~nm}$ thick film as hole blocking layer to avoid emission from Alq3. The hole transport layer (HTL) is a $20 \mathrm{~nm}$ thick film of polyvinylcarbazole (PVK, Aldrich Mw 1,100,000) deposited by spin-coating at $4500 \mathrm{rpm}$ for $40 \mathrm{~s}$ and dried at $150{ }^{\circ} \mathrm{C}$ for 5 minutes. The emitting layer $\mathrm{ZnO}: 3$ is spin-coated on the stack from concentrated nanohybrids solution in chlorobenzene $(20 \mathrm{mg} / \mathrm{mL})$. A $40 \mathrm{~nm}$ thick layer of Tris $(8-$ hydroxyquinoline)aluminum(III) (Alq3, Aldrich) is then used as electron transport layer (ETL). A thin layer of lithium fluoride ( $\mathrm{LiF}$ ) is used as electron injection layer (EIL) sequentially capped with a $100 \mathrm{~nm}$ thick layer of aluminum as cathode. $\mathrm{CuPc}, \mathrm{mCP}, \mathrm{Alq} 3, \mathrm{LiF}$ and $\mathrm{Al}$ layers were deposited at a rate of 0.2 $\mathrm{nm} / \mathrm{s}$ under high vacuum $\left(10^{-7} \mathrm{mbar}\right)$. The active area of the devices defined by the Al cathode was 0.3 $\mathrm{cm}^{2}$. After deposition, all the measurements were performed at room temperature under ambient atmosphere with no further encapsulation of devices. The current-voltage-luminance (I-V-L) characteristics of the devices were measured with a regulated power supply (ACT100 Fontaine) combined with a multimeter (Keithley) and a $1 \mathrm{~cm}^{2}$ area silicon calibrated photodiode (Hamamatsu). Electroluminescence (EL) spectra and chromaticity coordinates of the devices were recorded with a PR650 SpectraScan spectrophotometer, with a spectral resolution of $4 \mathrm{~nm}$.

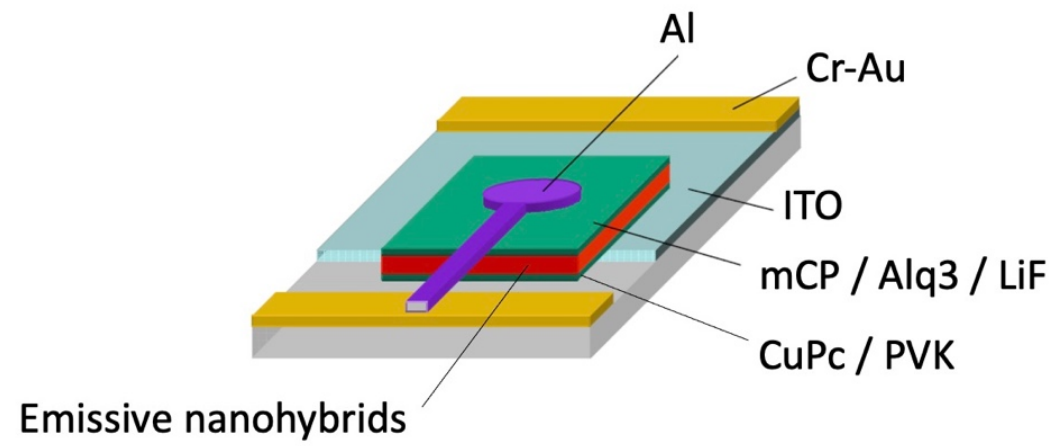

Figure S9: Scheme of the structure of the LED devices. 